\title{
Empirische Methode: Herleitung und Anwendung
}

Der zuvor herausgearbeitete Forschungsstand und die dargestellten theoretischen Vorüberlegungen zeigen, dass eine systematische Überprüfung zu Merkmalen und Bedingungen der Arbeitsgestaltung von Social Entrepreneuren in Deutschland bis dato nicht besteht, geschweige denn in wissenschaftlich abgesicherter Form. Demzufolge wird in diesem Kapitel für das Beantworten der zentralen Forschungsfrage ein qualitativ-empirisches Untersuchungsdesign als Interviewforschung hergeleitet und erläutert. Die angewandten empirischen Verfahren - von der Datenerhebung bis zur Datenauswertung - werden begründend ausgewählt und genau beschrieben.

Für das Beantworten der zentralen Forschungsfrage sind nicht einfach die persönlichen Einblicke, vorliegenden Hinweise und herausgearbeiteten Annahmen einzubinden, sondern Raum für das unvoreingenommene Entdecken neuer Erkenntnisse zu schaffen, denen sich sämtliche Präkonzepte unterzuordnen haben. Somit würde sich ein Formulieren von Hypothesen zu Beginn des Forschens als verfrüht erweisen, da das damit verbundene Fokussieren das ggf. noch Unbekannte nicht systematisch erfassen und einbeziehen kann, sondern eventuell sogar ausschließt. Folglich ist ein exploratives Forschungsdesign zu wählen. Als Fälle bieten sich Social Entrepreneure in Deutschland an, wobei bewusst zu reflektieren ist, wer (nicht) als „geeignet“ gelten darf oder gelten soll bzw. welche Bewertungskriterien heranzuziehen sind, um die Stichprobe so zielführend wie möglich zu ziehen bzw. die Fallauswahl zielführend zu gestalten. Ebenso sind die Bewertungsperspektive und die Indikatoren zur Bewertung offenzulegen. Denn das Forschungsfeld stellt die noch nicht systematisch erforschte Landschaft der

Elektronisches Zusatzmaterial Die elektronische Version dieses Kapitels enthält Zusatzmaterial, das berechtigten Benutzern zur Verfügung steht https://doi.org/10.1007/978-3-658-35145-8_3. 
Social Entrepreneure dar, wobei die Betroffenen zu ihrer Lebens- und Erfahrungswelt befragt werden sollen. Dabei darf das Untersuchen einzelner Fälle nicht per Zufallsstichprobe erfolgen, da das Aufdecken und Beschreiben möglichst aller relevanter Kriterien und Phänomene sichergestellt werden soll. Deswegen soll eine Systematik in der Datenerhebung gewährleisten, dass sich das Sampling theoretisch möglichst schlüssig abschließen lässt.

Schlussfolgernd kann für die vorliegende Studie nur ein qualitatives, induktives Forschungsdesign in Betracht kommen. Damit kann aus dem Erkenntnisgewinn kein Repräsentativitätsanspruch abgeleitet werden, aber ein Anspruch auf Reproduzierbarkeit der gewonnenen Daten. Herauszuarbeiten ist, inwieweit die Befragung theoretische Konzepte hervorbringt, die sich als Regelwerk abbilden lassen, um so die Merkmale und Bedingungen der Arbeitsgestaltung und eventuell die Wirkung von Arbeit von Social Entrepreneuren in Deutschland systematisch greifbar und begreifbar zu machen und hierbei eventuell sogar eine Typologie zu identifizieren.

Da die zur Beantwortung der Forschungsfrage relevanten Kriterien nicht als vollständig bekannt angenommen werden können, ist für die systematische Befragung der Bottom-up-Erhebungsansatz zu wählen. Gewährleistet wird damit, die bei der Datenerhebung schrittweise gewonnenen Erkenntnisse im Entwicklungsprozess im Feld hinsichtlich Konsistenz und Inkonsistenz zu spiegeln, zumal auf Basis der theoretischen Vorüberlegungen davon auszugehen ist, dass Social Entrepreneure sehr unterschiedliche und vielschichtige Lebensläufe vorweisen. Folglich ist ein Forschungsdesign zu wählen, das sowohl standardisiert abläuft, i. S. v. empirisch abgesichert ist, als auch hinreichend Flexibilität bei der Datengenerierung und -analyse zulässt, bei dem für den Prozess des Erkenntnisgewinns das Postulat der theoretischen Offenheit befolgt werden kann. Datenerhebung und Datenauswertung wachsen dabei parallel und kontinuierlich. Sie werden in ihrer wechselseitigen Entwicklung miteinander verwoben und sollen sich produktiv beeinflussen (vgl. Strübing, 2014, S. 461 f.), so dass daraus eine stabile Struktur im permanenten Datenvergleich hervorgeht, vergleichbar mit drei regelhaft verknüpften Strängen, die einen Stabilität und Halt generierendes „Erkenntnisgeflecht" ausbilden. Dementsprechend erfolgt eine in sich schlüssige komparative Analyse aller emergierten Daten, bei der sich der Sozialforscher nach seiner ersten Auswertung zurück in sein Forschungsfeld begibt, um systematisch weitere Daten zu erheben und zu integrieren. Inwieweit im Rahmen der Datenauswertung und -interpretation weitere Verfahren hinzugezogen und auf Teilfragestellungen bezogen oder auch hinsichtlich einzelner Fälle angewandt werden sollen, ist im Auswertungsprozess anforderungsspezifisch zu entscheiden. Dies erfordert nicht 
nur das konsequente Befolgen ausführlicher Transkriptionsregeln, die das Integrieren verschiedenartiger interpretativer Verfahren gewährleisten, sondern vor allem das Anwenden einer übergeordneten empirischen Methode, die es erlaubt, unterschiedliche Ansätze zur Datenauswertung bedarfsorientiert zu integrieren.

Sämtliche beschriebenen empirischen Voraussetzungen lassen sich vereinbaren mit der Methodologie der ,Reflexiven Grounded Theory“ (RGT) nach Breuer (1996; 2010) sowie Breuer, Muckel und Dieris (2017; 2019). Demzufolge basiert die vorliegende Untersuchung auf diesem Forschungsverfahren.

\subsection{Reflexive Grounded Theory (RGT) als übergeordnete empirische Methode}

Bei der RGT handelt es sich um eine Ausdifferenzierung des ursprünglich von Glaser und Strauss (1967) entworfenen und beschriebenen Forschungsansatzes „Grounded Theory“. Zielsetzung von Glaser und Strauss ist es, eine wissenschaftlich legitimierte Vorgehensweise zum Aufdecken und Herausarbeiten von Theorien aus Datenmaterial zu generieren bzw. bestehende Theorien konstruktiv infrage stellen zu können. Damit schaffen die Autoren einen Gegenpol zur damals funktionalistisch geprägten, quantitative Survey-Studien fokussierenden Forschung (ebd., vgl. auch Legewie \& Schervier-Legewie, 1995, S. 70 f.; Strauss, 1991, S. 39 f.) und setzen ,direkt am Positivismusstreit rund um die Frankfurter Schule an, die dem empirisch-analytischen (positivistischen) Ansatz ein deterministisches Menschenbild sowie triviale Arbeitsergebnisse vorwirft, da sie mit dem Herausgreifen vereinzelter Variablen die soziale Komplexität verkennt, wodurch ein Rekonstruieren von Sinn und das Abbilden von Bedeutungszusammenhängen per se ausgeschlossen ist" (Hein, 2016, S. 170).

Allerdings beinhaltet der von Glaser und Strauss (1965; 1967) formulierte Grundstein zur qualitativen Auswertungsanalyse noch keine Regelhaftigkeit mit How-to-do-Charakter, sondern die konzeptionelle Ausgestaltung konzentriert sich zunächst auf das Erläutern eines Basisverständnisses auf der Haltungsebene. Das damit mangelhaft vorliegende Konzeptverständnis lässt sich im direkten Vergleich zum - wenngleich der pädagogischen Arbeit entstammenden, aber im Kern dennoch auf empirisches Arbeiten übertragbaren - Konzeptbegriff nach Reiser und Lotz (1995) aufzeigen: Sie unterscheiden zwischen (1) einer das Menschenbild beschreibenden Theorieebene, (2) einer darauf basierenden handlungsrichtungsweisenden Methodenebene sowie (3) einer wiederum hierauf basierenden, regelgeleitet-präzisierenden sowie Handlungsanweisungen verkörpernden Technikebene. Glaser und Strauss (1967) verbleiben auf der das Menschenbild 
beschreibenden Theorieebene und zeigen dabei eine Affinität zu den Ausführungen des symbolischen Interaktionismus auf: Der Bedeutungsgehalt sozialer „Objekte“ und deren Beziehungen zueinander emergieren aus einem symbolisch vermittelten kommunikativen Interaktionsprozess. Glaser positioniert sich dabei zunehmend als Vertreter bedingungsloser Emergenz und negiert Apriori letztendlich uneingeschränkt (2012; vgl. zuvor bereits in Glaser \& Holton, 2011; Glaser \& Tarozzi, 2011), im Gegensatz zu Strauss (1991), was zum Bruch der Zusammenarbeit zwischen den beiden Sozialforschern führt. Hierbei entwickelt Strauss (ebd.), später gemeinsam mit Corbin (1996), eine Kodiermethodik, die bereits auf der konzeptionellen Theorieebene ausdrücklich einfordert, Apriori einzubeziehen. Fortan grenzen sich Strauss und Corbin auch begrifflich von Glasers Arbeitsweise ab, indem sie ihren Forschungsansatz als „Grounded Theory Methodology“ (GTM) bezeichnen. Deren Kodiermethodik beinhaltet zudem eine konzeptionelle Methoden- wie auch Technikebene, wobei sich sämtliche Ausführungen mit der RGT-Methodologie vereinbaren lassen, sofern sie eine intensive, auf den kompletten Forschungsprozess bezogene methodenkritische Reflexion beinhalten, die sich in der Darstellung von Forschungsergebnissen widerspiegelt. Denn von zentraler Bedeutung ist in der Reflexiven GT, dass jeder Teilschritt eines Forschungsprojekts stets situativ durch die Einflussnahme des qualitativen Sozialforschers im kontinuierlichen Entwicklungsprozess entsteht. Wissenschaftliche Erkenntnisse stehen also stets in Abhängigkeit zu der Person, die diese Erkenntnisse hervorbringt. Damit wird der Forscher zum „Forscher-Subjekt“ und der Erforschte wird zum „Forschungs-Subjekt“ (Breuer, 2018; Breuer et al., 2019, S. 84; vgl. Charmaz, 2001; als frühe, indirekt formulierte Forderung bei Robson, 1993, S. 242 f., 460; ausführlicher in 2002, z. B. S. 163 ff.). Der sich daraus ergebende, bedeutsame Reflexionsanspruch erklärt dessen Verankerung im Namen dieses Forschungsverfahrens.

Die in der vorliegenden Untersuchung praktizierte Datenerhebung, Datenaufbereitung und Datenauswertung wird nachfolgend genau beschrieben, inklusive einer ausführlichen methodenkritischen Reflexion für alle Forschungsschritte. Abbildung 3.1 zeigt zur forschungsprojektbezogenen Qualitätssicherung den Forschungsprozessablauf als Workflow. Eine tabellarische Übersicht zum chronologischen Verlauf der durchgeführten Aktivitäten sichert die intersubjektive Nachvollziehbarkeit zusätzlich und ist im elektronischen Zusatzmaterial im Anhang B wiedergegeben. 


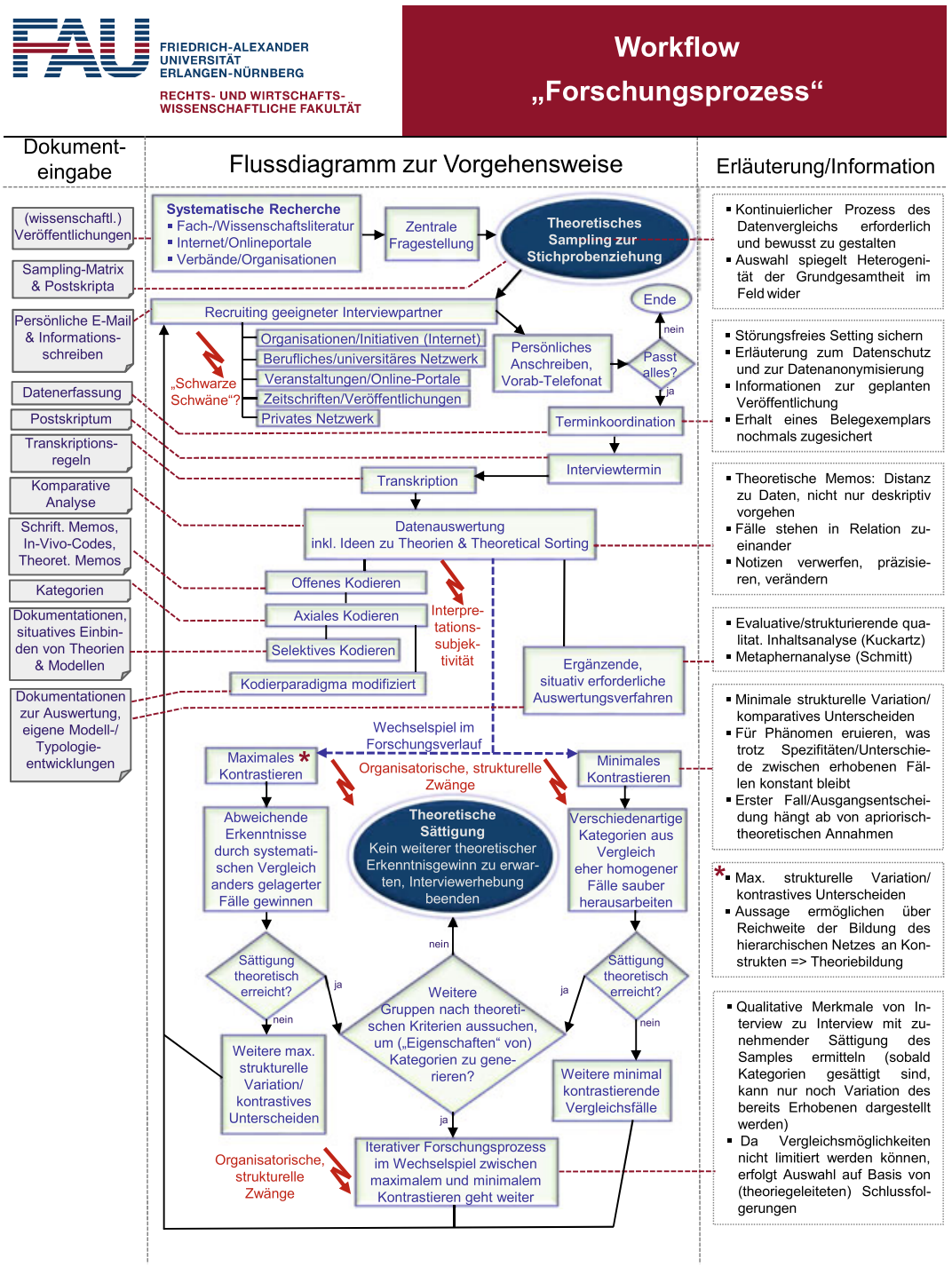

Abbildung 3.1 Forschungsprozessablauf als Workflow. (eigene Darstellung) 


\subsection{Datenerhebung}

Nachdem die grundlegenden Anforderungen an das Forschungsdesign hergeleitet worden sind, ist dieses nun zu präzisieren - beginnend mit der Datenerhebung. Als passgenaue Erhebungsmethode eignet sich die Form eines Einzelinterviews. Dabei werden die Anforderungen an die Vorbereitung, Durchführung und Nachbereitung herausgearbeitet.

\subsubsection{Problemzentriertes Interview mit narrativen Elementen}

Für das im lückenhaften Forschungsstand begründete explorative Design wird eine Erhebungsmethode benötigt, die den Befragten mit Offenheit begegnet und dabei den Einbezug von Vorerkenntnissen gewährleistet. Die Befragten sollen dabei hinreichend Vertrauen generieren können, damit sie ihre Beweggründe und Ansichten bestmöglich reflektieren und ihre Gedanken hierzu auszusprechen können, wobei Ausführungen der Befragten zu unerwarteten, vom Forscher in seiner Interviewplanung nicht bedachten Aspekten ausdrücklich erwünscht sind. Somit soll die anzuwendende Interviewform einen Zugang zur Innensicht der Untersuchungsteilnehmer schaffen und die Befragten motivieren können, gesellschaftlich wie biografisch relevante Problemstellungen im Hinblick auf ihre eigenen lebensgeschichtlichen Hintergründe zu reflektieren, ihre Handlungsmotive zu erläutern sowie prägende Momente zu gesellschaftlichen Entwicklungen in Erinnerung zu rufen und verbalisieren zu können. Ebenso soll die Möglichkeit bestehen, Rückfragen durch den Interviewer zuzulassen. Grundsätzlich eignen sich hierzu leitfadengestützte Einzelinterviews (vgl. Przyborski \& Wohlrab-Sahr, 2014, S. 126 ff.; Helfferich, 2011), wobei das aufgezeigte Anforderungsprofil mit dem leitfadengestützten ,problemzentrierten Interview“ optimal vereinbar erscheint (vgl. Flick, 2017, S. 210 ff.; Witzel, 1985; 2000; Hussy, Schreier \& Echterhoff, 2013), dies unter gezieltem Einbezug von Elementen des narrativen Interviews (nach Schütze, 1977). Diese beiden Interviewformen lassen sich sinnvoll kombinieren (vgl. z. B. Mey, 2000; Helfferich, 2011, S. 13 ff.). So erlaubt es der Aufforderungscharakter des problemzentrierten Interviews dem Forscher beispielsweise, beim abschließenden Betrachten einer Problemstellung oder auch zum Gesprächsabschluss weitere, vom Interviewpartner nicht angesprochene Aspekte in offener Fragestellung und ohne Suggestivelemente einzubringen, die in bereits geführten Interviews zum Relevanzsystem der ehemals Befragten zu verorten sind oder präkonzeptionell bedeutungsvoll erscheinen. Der Einbau narrativer Elemente ermöglicht es dem Forscher, das Gespräch ergebnisoffen zu 
gestalten und den Befragten ab Interviewbeginn, ohne inhaltlich einzuschränken, zum Erzählen aufzufordern. Auf diese Weise lassen sich etwaige weitere Sinnzusammenhänge herausarbeiten. Dabei darf der Interviewer keinesfalls Meinungen abfragen, sondern hat rezeptiv aufzutreten und soll sich durch die Erfahrungswerte des Befragten regelrecht belehren lassen (ebd.), was sich in der Gestaltung des Interviewleitfadens widerzuspiegeln hat.

\subsubsection{Interviewleitfaden}

Das problemzentrierte Interview beinhaltet methodische Standardisierungselemente bei technisch geringem Strukturierungsgrad. Vorbereitete Fragen werden an die jeweilige Gesprächssituation angepasst und sollen zum freien Erzählen auffordern, wobei der Befragte seiner eigenen, inneren Gedankenstruktur treu bleiben soll. Dementsprechend gleicht der Leitfaden einer interviewspezifischen Skizze, die eine zu erforschende Themenlandschaft andeutet, ohne sie zu sehr einzugrenzen. Dabei ist Offenheit zu wahren, um neue Themen aufgreifen oder Fragen in geänderter Reihenfolge stellen oder teilweise auch vernachlässigen zu können. Demzufolge lassen sich bei der Entwicklung eines Folgeleitfadens aus dem bisherigen Leitfadengerüst diejenigen Fragestellungen, die in bereits geführten und ausgewerteten Interviews ausführlich beantwortet worden sind, verändern bzw. entfernen zugunsten des Integrierens neu aufgedeckter, bislang unbeantworteter Fragestellungen.

Beim Verfassen aller Interviewleitfäden wurde bei der Darstellungsform der Praktikabilität halber darauf geachtet, dass sämtliche Versionen einseitig sind, in thematischen Blöcken strukturiert vorliegen und eine innere Logik für vielseitig denkbare, alternative Gesprächsverläufe unter der Einbindung von Ad-hoc-Fragen aufweisen. Anhang C im elektronischen Zusatzmaterial zeigt beispielhaft den Leitfaden für IP10. Dieser thematisiert zunächst das eigene SE-Verständnis, den eigenen Werdegang, erste Erfahrungswerte und den Stellenwert von Vernetzung, fokussiert im mittleren Teil Herausforderungen und geht abschließend auf Qualifikation und Kompetenzen ein. Die innere Struktur einer solchen „Leitfadenskizze“ ist für Dritte mit hoher Wahrscheinlichkeit nicht erschließbar, zumal die eigentlichen Leitfragen als Hilfestellungen, beispielsweise in Stichwörtern oder als Mindmap, skizziert werden können. Dies schmälert keinesfalls die Bedeutung und Qualität des Interviewleitfadens, sondern soll sogar und kann eine qualifizierte, passgenaue Gesprächsvorbereitung und -durchführung sicherstellen, zumal für den Interviewer stets eine Übersichtlichkeit gegeben sein muss. In diesem Sinn können sortierte und gruppierte Stichwörter sowie das Verwenden von 
Symbolen gewährleisten, keine Fragen abzulesen, sondern sie situativ-passgenau und deutlich besser im Augenkontakt zum IP verbleibend zu formulieren. Beim nochmaligen Anhören eines Interviews und auch dem späteren Lesen einer vollständigen Interviewtranskription lässt sich, wenngleich begrenzt, feststellen, inwieweit ein Leitfaden seinen Zweck erfüllt hat.

Als inhaltliche Basis dienen zu jeder Gesprächsvorbereitung der aktuell erhobene Ist-Stand zur komparativen Analyse im Prozess des theoretischen Samplings unter Einbezug aller weiteren Erkenntnisse und Fragestellungen, die z. B. mit der IP-spezifischen Recherche vorliegen. Dabei dienen sämtliche Vorerkenntnisse nicht zum Abfragen, sondern sie werden bei der Leitfadengestaltung als Präkonzepte berücksichtigt, die zum Reflektieren der eigenen Rücksichtnahme im Forschungsfeld dienen und ein ergebnisoffenes Befragen wahren. Unabhängig davon, dass die dargestellten qualitativen und quantitativen Studien offensichtlich nur eingeschränkt konsistente sowie in der Genese zum Teil ungesicherte Erkenntnisse widerspiegeln, prägen sie Vorannahmen beim eigenen Forschen und ermöglichen das Generieren geeigneter Fragestellungen.

\subsubsection{Theoretisches Sampling, Fallkontrastierung und theoretische Sättigung}

Zur Absicherung ökologischer Validität ist bei der Fallauswahl darauf zu achten, dass sie der Alltagsrealität der Untersuchungssituationen entspricht. Glaser und Strauss (1998, S. 53 ff.) prägten den Ansatz der theoretischen Stichprobenziehung im Sinne einer absichtsvoll gestalteten Fallauswahl im Kontext ihrer Grounded-Theory-Entwicklung. Die getroffene Fallauswahl spiegelt hierbei die Heterogenität des gesamten Forschungsfelds wider. Normativ hat die Entscheidung für einen Interviewpartner jeweils nach der Datenauswertung des vorherigen Interviews zu erfolgen. Lediglich die erste Fallauswahl erfolgt zwangsläufig willkürlich. Bei dieser Studie handelt es sich dabei um das Kriterium „als Social Entrepreneur gescheitert“.

Basierend auf dem Erkenntnisgewinn, der sich aus der Datenauswertung des vorherigen Interviews ergibt, soll sich bewusst für eine minimale oder maximale Fallkontrastierung entschieden und aus der daraus abgeleiteten Kontrastierungsrelevanz ein Termin mit einem geeigneten Folge-Interviewpartner vereinbart werden. Hat das Auswerten eines Interviews zu einem untersuchten Kriterium neue Erkenntnisse hervorgebracht, kann im darauffolgenden Interview dieser neu identifizierte Erkenntnisaspekt mit einem vergleichbaren Fall vertieft werden. Dies 
entspricht dem minimalen Kontrastieren, z. B. realisiert durch das Erforschen verschiedener Erfahrungswerte im Umgang mit dem Scheitern. Erst wenn sich kein neuer Erkenntnisgewinn aus einem minimal kontrastierten untersuchten Kriterium generieren lässt, sollte eine entgegengesetzte Kriterienausprägung fokussiert werden. Dies wiederum entspricht dem maximalen Kontrastieren, z. B. realisiert durch das Erforschen von besonders erfolgreichen, nicht ansatzweise gescheiterten Social Entrepreneuren. Auf diesem Weg lassen sich Auswahlkriterien im Forschungsprozess herausarbeiten und aufeinander aufbauend entwickeln. Das Kontrastierungsverfahren stößt jedoch aus mehreren Gründen an seine Grenzen. Zum einen können mehrere Kriterien gleichwertig relevant erscheinen: beispielsweise die Kombination aus „Gescheitert sein“ und ,langjährige Erfahrung als SE“. Auch in der vorliegenden Untersuchung ergibt sich ein zunehmend komplexes, multidimensionales Bedingungsgefüge an Einflusskriterien, die bei Social Entrepreneuren in unterschiedlicher Kombination und Intensität bedeutungsvoll erscheinen. Somit ist eine lineare Fallkontrastierung per se nicht abbildbar. Einzelne Kriterien müssen zur Kontrastierung priorisiert werden - zulasten weiterer Kriterien, die demzufolge zu einem anderen Zeitpunkt im Datenerhebungsprozess im Mittelpunkt zu stehen haben. Insofern ist ein willkürliches Eingreifen unabdingbar. Zum anderen würde sich der Forschungsprozess unverhältnismäßig in die Länge ziehen, wenn bei der Interviewterminierung die durch die Interviewpartner vorgegebenen Restriktionen wie Terminverschiebungen und mehrmonatige Wartezeiten den Ablauf dominieren (vgl. auch Strübing, 2014, S. 465). Zu bedenken ist ebenfalls, dass Zwischenergebnisse lediglich den Stellenwert von Vorannahmen für den gegenwärtigen Forschungszeitpunkt innehaben können.

Umso wichtiger erscheint, im Rahmen der Fallkontrastierung eine Technik anwenden zu können, die es erlaubt, sämtliche Kriterien nicht aus dem Blick zu verlieren, sondern die sie im Gesamtkontext als gleichwertig betrachtet und eine theoretische Sättigung dennoch gewährleistet. Dabei ist zu berücksichtigen, dass, wie kapiteleinleitend ausgeführt, neue relevante Kriterien erst im Laufe des Erhebungsprozesses aus dem Datenmaterial hervorgehen können, so dass bereits ausgewertete Interviews hinsichtlich einer neuen Erkenntnis so gut wie möglich abermals auszuwerten sind. Die entsprechende Fallauswahl-Datei, die zur Wahrung der Anonymisierung der Befragten nicht mit veröffentlicht werden kann, zeigt, wie in der vorliegenden Untersuchung mit der damit formulierten Herausforderung umgegangen wird. Der Lösungsansatz ist eine Sampling-Matrixstruktur, die sämtliche im Verlauf der Datenerhebung berücksichtigten Kriterien als gewachsene Struktur abbildet. Weiterführende Ausführungen und Reflexionen, die für das Bestimmen geeigneter Folgeinterviewpartner von Bedeutung sind, werden in den Postskripta dokumentiert, die, ebenso wie die 
Interviewtranskriptionen, zur Wahrung der Anonymisierung der Befragten nicht mit veröffentlicht werden können. Einen Steckbrief zur Fallauswahl zeigt Anhang D im elektronischen Zusatzmaterial.

Der Befragungsprozess hat so lange zu erfolgen, bis kein weiterer Erkenntnisgewinn mehr generiert werden kann und dies, mit dem angewandten SamplingVerfahren bestmöglich abgesichert, auch nicht mehr zu erwarten ist. Damit ist die Grundvoraussetzung geschaffen, eine theoretische Sättigung unterstellen zu können. Dies ist bei der vorliegenden Untersuchung mit dem 34. Interview gegeben. Das 35. Interview lässt sich dennoch als sinnvolles „Kontrollinterview“ bewerten. IP35 stand erst mehrere Monate nach Terminanfrage für das Interview zur Verfügung, bevor die letzten Interviewtermine vereinbart wurden. Das Interview mit IP35 hat keinen neuen Erkenntnisgewinn hervorgebracht, womit eine theoretische Sättigung begründet wird.

Nachfolgend wird beschrieben, wie das theoretische Sampling für alle Interviewpartner konkret gestaltet wird.

\subsubsection{Interviewpartner-Recherche, Kontaktaufnahme und Terminkoordination}

Zur Rekrutierung geeigneter Interviewpartner dienten bei der vorliegenden Studie eine intensive Fachzeitschriften-, Fachbücher- und Internetrecherche, der Zugriff auf private, berufliche und universitäre Netzwerke sowie im Bundesgebiet regional und überregional vertretene Events und Tagungen. Mehrfach boten befragte Social Entrepreneure im direkten Anschluss an deren Interview an, ihr eigenes Netzwerk gezielt für eine Kontaktvermittlung zu aktivieren. Dies wurde im Laufe des Datenerhebungsprozesses in drei Fällen in Anspruch genommen, bei denen die potenziellen Interviewpartner zur geplanten Fallauswahl passten. Für das Weiterleiten der Interviewanfrage innerhalb der Netzwerke von angefragten Personen und Institutionen wurde ein Informationsschreiben zur Verfügung gestellt, das neben der Kurzbeschreibung des Dissertationsprojekts die Zusicherung der Datenanonymisierung sowie die Zusicherung des Erhalts eines Exemplars der Studie enthält. Das Informationsschreiben wurde im Laufe des Datenerhebungsprozesses zweimal angepasst. Anhang E im elektronischen Zusatzmaterial zeigt exemplarisch eine der drei Versionen.

Jede direkte Kontaktaufnahme zu einem potenziellen Interviewpartner erfolgte mit persönlicher Ansprache in einer E-Mail, der eines der zuvor dargestellten Informationsschreiben beigefügt ist und in der eine telefonische Kontaktaufnahme angekündigt wird, um für Rückfragen persönlich zur Verfügung zu stehen, 
die Passgenauigkeit als Interviewpartner noch einmal zu überprüfen und einen Interviewtermin im möglichst störungsfreien Setting zu koordinieren.

Bei der Terminkoordination wurde darauf geachtet, dass das Interview im persönlichen, analog gestalteten, direkten Kontakt stattfindet, bei dem möglichst störungsfrei und in Ruhe über Herausforderungen, die der Befragte als Social Entrepreneur in seiner Arbeitsgestaltung erlebt, gesprochen werden kann. Die Notwendigkeit des Interviews im Vis-à-vis-Kontakt wurde bedarfsorientiert erläutert - z. B. wenn ein Social Entrepreneur darum bat, dass das Interview per Video durchgeführt wird. Ebenso wurden das elektronische Aufzeichnen der Gespräche und der gewissenhafte Umgang mit den Daten erläutert.

Die Interviewpartner favorisierten unterschiedliche Treffpunkte: zumeist ihren eigenen Arbeitsplatz und deutlich seltener einen vom Interviewer zu organisierenden Besprechungsraum. In zwei Fällen boten hochfrequent geschäftlich reisende IP lediglich einen vorgegebenen, dennoch hinreichend langen Gesprächsslot in einem ihnen vertrauten Café während einer Dienstreise an.

\subsubsection{Erhebungszeitraum und Datenaufzeichnung}

Die 35 Interviews wurden ab KW 12/2018 bis KW 39/2019 durchgeführt. Die durchschnittliche Gesprächsdauer beträgt>90 min. Das kürzeste Gespräch dauert 46 min, das längste 144. Bis auf drei Gespräche dauern alle länger als eine Stunde. Bei zeitlich relativ eng getakteten Interviews wurde sichergestellt, dass zwischen den Gesprächen eine Ausarbeitung an Memos und erste Kodierungen vorgenommen werden konnten, ebenso eine Nachbereitung und weitere Planung des theoretischen Samplings.

Um den nicht immer gut kalkulierbaren Aufnahmebedingungen gerecht zu werden, die z. B. durch sporadisch störende Hintergrundgeräusche bei wechselhaft lauter Sprechweise oder durch prosodische Unregelmäßigkeiten seitens der Befragten gegeben sind und die Aufnahmequalität beeinträchtigen, wurden alle Interviews mit drei professionellen Diktiergeräten in unterschiedlichen Einstellungsmodi im MP3-Kodierformat aufgezeichnet. Bei den Geräten handelt es sich um die Modelle VN 733 von Olympus sowie DVT 2000 und DVT 3500 von Philips. Damit ist für sämtliche Interviewsequenzen mit mindestens einem Aufnahmegerät eine gut verstehbare Wiedergabe sichergestellt, was die Ausgangsbedingung für ein fehlerfreies und möglichst lückenloses Transkribieren darstellt.

Neben den elektronisch aufgezeichneten Informationen trafen die Befragten weitere forschungsfragenrelevante Aussagen: in Vorabtelefonaten, im direkten 
Anschluss an das Ausschalten der Aufnahmegeräte, in Nachtelefonaten oder auch per E-Mail-Kontakt. Diese wurden in Postskripta dokumentiert sowie in Memos, die direkt in das softwaregestützte Datenmaterial eingepflegt werden.

Über die 35 Interviews hinaus wurden fünf Fachgespräche mit Vertretern von SEO-Unterstützungsorganisationen geführt. Die Fachgespräche dienen zur klärenden Beantwortung diverser Fragestellungen und wurden daher nicht komplett kodiert, sondern unterstützend herangezogen; sie fanden zwischen KW 13/2019 und KW 37/2019 statt. Deren Datenaufzeichnung und -dokumentation erfolgte auf gleiche Weise wie bei den Interviews.

\subsubsection{Interviewablauf und Gesprächsgestaltung}

Vor dem Einschalten der Aufnahmegeräte wurde der zugesicherte Schutz der Daten inkl. Anonymisierung noch einmal bekräftigt und der Dank für die Teilnahme an der Befragung bekundet. Rückfragen der IP wurden ggf. beantwortet. Sofern Fragen für das Beantworten der zentralen Forschungsfrage relevant erschienen, wurde um Erlaubnis für das Einschalten der Aufnahmegeräte gebeten und anschließend mit dem Interview begonnen.

Aus den Axiomen zur Kommunikation von Watzlawick, Beavin und Jackson (1990) lässt sich ableiten, dass der Interviewer sich gegenüber seinem Interviewpartner „nicht nicht verhalten“ kann. Interviewer intervenieren in ihrem Forschungsfeld und wirken agierend und reagierend durch ihr Verhalten und ihre Kontaktgestaltung auf ihre Interviewpartner ein - und umgekehrt (ebd., S. 50 ff.). Dabei wird in der Interviewforschung ein persönlicher Face-to-face-Kontakt zwischen Forschendem und Erforschtem favorisiert, um Verzerrungseffekte in der Kommunikation möglichst zu vermeiden bzw. zu reduzieren.

Zum Gesprächseinstieg diente bei allen Interviews eine Frage, die vom akademischen Anspruchsniveau ablenken und den Effekt sozialer Erwünschtheit unterbinden soll. Diese lautet sinngemäß: „Wenn wir davon ausgehen, dass es kein Richtig und kein Falsch gibt, sondern nur die eigene, ganz persönliche Meinung: Was bedeutet für Sie ganz persönlich Sozialunternehmertum oder auch Social Entrepreneurship?“

Die Abschlussfrage lautete in jedem Interview sinngemäß: „Wenn Sie daran denken, welche Merkmale oder Bedingungen Sie als Social Entrepreneur bei Ihrer Arbeit erleben, inwieweit haben wir bestimmte Punkte dazu noch gar nicht angesprochen?" Damit soll sichergestellt werden, dass bislang unberücksichtigt gebliebene Aspekte thematisiert werden können. 
Für den Fall, dass bestimmte demografische Daten, die für die Datenauswertung relevant erscheinen, bis zum Gesprächsabschluss noch nicht bekannt geworden sind, wurden diese abschließend erfragt - entweder vor oder auch nach dem Ausschalten der Aufnahmegeräte. Bewusst wird das Abfragen zu Beginn des Interviews vermieden, da dies zum einen das Prinzip der Offenheit gefährden kann; denn Gedanken zu Rückschlüssen auf die eigene Person können störend einwirken und den Erzählfluss insbesondere von Belastungssituationen gefährden. Zum anderen geben IP von sich aus während des Interviews erfahrungsgemäß demografische Daten preis, was ein Nachfragen überflüssig macht.

Nach den Interviews erhielten die IP ein zuvor nicht angekündigtes Präsent mit einem Dankes-Begleitschreiben, in dem abermals zugesichert wird, dass sie ein Exemplar der Ausarbeitung erhalten werden. Das Begleitschreiben wurde aufgrund einer Variation im Präsent einmal angepasst; im elektronischen Zusatzmaterial im Anhang F befindet sich beispielhaft eine der beiden Versionen.

Im Anschluss an die Beschreibung der Planung und Durchführung der Datenerhebung erfolgt nun eine methodenkritische Reflexion, bei der speziell auf die Gütekriterien qualitativer Forschung eingegangen wird.

\subsubsection{Methodenkritische Reflexion zur Datenerhebung}

Die Ausführung zur Gesprächsgestaltung weist bereits darauf hin, dass ab der Datenerhebung qualitätssichernde Maßnahmen zu treffen sind. Dabei sind im Hinblick auf das Bewerten der Güte vielseitige Einflusskriterien zu berücksichtigen, die im Folgenden besprochen werden. Allerdings besitzen die quantitative und die qualitative Forschung keine gemeinsame erkenntnistheoretische, methodologische Basis für Gütekriterien. Demzufolge können Gütekriterien quantitativer Forschung keine orientierungsweisende Funktion für die vorliegende Studie darstellen. Die Reflexion des subjektiv begründeten Vorgehens des Forschers ermöglicht es, daraus Konsequenzen für die Dateninterpretation abzuleiten, Grenzen der Arbeit aufzuzeigen und zugleich die Güte der Erkenntnisse zu bewerten.

Gemäß der bereits erfolgten Darstellung gehört es zum Selbstverständnis der RGT, jeden qualitativen Forschungskontakt als soziale Interaktion anzusehen (vgl. Breuer et al., 2017, S. 77) und den Forscher somit als Forschersubjekt zu verstehen (ebd., S. 234; vgl. Breuer, 1999, S. 264 ff.). Empfehlenswert erscheint dabei, die methodenkritische Reflexion nicht in der für empirische Arbeiten sonst üblichen, quasi-objektivierenden dritten Person Singular zu verfassen, sondern in der Ich-Form, um die Gefahr zu reduzieren, durch sprachlich künstlich geschaffene Distanz Verzerrungseffekte einzubauen (vgl. Breuer, 2018). Dies werde ich 
nun praktizieren. Auf gleiche Art und Weise werde ich in Abschnitt 3.3.3 zur methodenkritischen Reflexion der Datenaufbereitung und in Abschnitt 3.4.4 bei der Datenauswertung verfahren.

Darüber hinaus formuliere ich forschungsprojektbezogene Handlungen im Präteritum und nutze das sonst übliche generelle Präsens vor allem bei der Darstellung des Ablaufs der Befragung und der zeitübergreifend gültigen Ergebnisse, um keinen Interpretationsspielraum für allgemeingültige Aussagen zu schaffen.

\section{Supervision und Arbeitsgruppen}

Die Interviews führte ich ohne Einbezug weiterer Interviewer durch. Aufgrund der hierbei fehlenden Forscherperson-Triangulation habe ich dennoch den Anspruch, die Vorbereitung, Durchführung und Nachbereitung der Datenerhebung intersubjektiv nachvollziehbar zu reflektieren, um beispielsweise Bias und Effekte, welche die Datenqualität beeinträchtigen können, zu vermeiden. Diese können sich z. B. bereits durch die Prägungen in meiner Biografie ergeben. So kann ich mich zwar einerseits mit meinem Hintergrund als Wirtschaftswissenschaftler mit dem Schwerpunkt Sustainability Management sowie als Arbeits- und Organisationspsychologe meinem Schnittstellenthema aus wissenschaftstheoretischer Perspektive mit interdisziplinärem Grundverständnis annähern, zumal ich neben meiner Lehrtätigkeit auch als qualitativer Sozialforscher im Bereich Arbeitsbedingungen und Arbeitsgestaltung mit wirtschaftsethischem Schwerpunkt erfahren bin. Als hilfreich sehe ich ebenfalls meine Mediatoren-Qualifikation sowie meine vorherige psychotherapeutische Berufsausübung an, bei der ich langjährig reflektiert habe, mir in beruflichen personenzentrierten Gesprächen meiner Meinung zwar bewusst zu sein, diese aber nicht als solche einzubringen, geschweige denn sie zu fokussieren. Andererseits prägen mich genau die Erfahrungswerte zur eigenen Meinungsbildung bezüglich Social Entrepreneurship, so z. B. meine Gründungs- und Vorstandstätigkeit bei einem gemeinnützigen Bildungsverein mit der Zielsetzung einer nachhaltigen Entwicklung für Gesellschaft, Umwelt und Wirtschaft, aus dem ein Sozialunternehmen hervorgegangen ist, das ich in seiner Entwicklung nah und intensiv beobachte und begleite.

Aus diesem Zwiespalt heraus habe ich mich entschieden, ab der Datenerhebungsphase bedarfsorientiert und anlassbezogen mehrere Kodier-Arbeitsgruppensettings auch zur Selbstreflexion zu nutzen und darüber hinaus bedarfsorientiert Einzelsupervisionssitzungen bei einer entsprechend geschulten Fachkraft in Anspruch zu nehmen. Mit meinem Einfordern von Feedback konnte ich Übertragungseffekte herausarbeiten, Interviews bestmöglich vor- und nachbereiten und ein „mentales Resetten" zum Mich-Einlassen auf einen neuen IP mit dessen eigenen Erfahrungswerten ermöglichen. Bei aufgedeckter Uneindeutigkeit in meiner Befragung konnte 
ich noch einmal auf einen IP zugehen und eine zur Klärung beitragende Rückfrage stellen, was sich positiv auf eine gewissenhafte Datenauswertung auswirken sollte. Darüber hinaus wandte ich unmittelbar vor nahezu jedem Interview eine mir bereits aus anderen Studien bekannte Entspannungsübung an, die mir dazu verholfen hat, so unvoreingenommen wie möglich in ein Interview ,einzusteigen“ (vgl. Hein, 2016, S. 186).

Die meisten IP verbalisierten tiefgründig emotionale Erlebnisinhalte und wirkten dabei kongruent und echt in ihren Schilderungen. Entweder während des Interviews oder direkt danach verglichen mehrere IP das Befragen mit einer psychotherapeutischen Sitzung (IP22 $2^{12} ; \mathrm{IP}^{13}$ ). Mehrfach betonten einige IP, dass sie das Interviews als ausgesprochen bereichernd empfunden haben und bereits zum Gesprächsabschluss merkten, dass sie aus dem Interview für sich etwas mitnehmen können. Dies gaben auch mehrere IP im Nachhinein per E-Mail und einmal im Telefonat von sich aus als persönliches Feedback. Bezüglich dieser Rückmeldungen konnte ich aufgrund meiner psychotherapeutischen Qualifikation und beruflichen Prägung eine fehlende Rollenabgrenzung durch mich nicht ausschließen, was die Professionalität der wissenschaftlich fundierten Interviewerhebung beeinträchtigen könnte. Daher habe ich auch hierzu Supervisionssitzungen und Arbeitsgruppentreffen genutzt und mein eigenes Wirken in der Rolle des Interviewers rückblickend reflektiert. Nähere Angaben zu den Mitgliedern der Arbeitsgruppen sind in der ChronologieSkizze zu Aktivitäten dargestellt (Anhang B im elektronischen Zusatzmaterial). Innerhalb dieser Treffen arbeiteten wir heraus, dass es mir mit der Befragung gelungen ist, die Zielsetzung des problemzentrierten Interviews mit narrativem Anteil zu erreichen, also in die Tiefe gehend und auf vertrauensvoller Gesprächsbasis mit Social Entrepreneuren Merkmale und Bedingungen zur Arbeitsgestaltung und deren Wirkung zu ergründen. Meine gesprächs- und gestalttherapeutische Erfahrung, gerade das Anwenden personenzentrierter Gesprächsführungstechniken nach Rogers (1985), erwies sich für das Ergründen neuer Erkenntnisse als hilfreich, nicht aber als hinderlich, da es mir beispielsweise relativ leicht gefallen ist, hinreichend Gesprächspausen während der Schilderungen von Belastungssituationen zuzulassen, anstatt neue Fragen zu stellen, was in der Folge keinen Themenbruch provoziert, sondern zum Ausführungen der subjektiven Erfahrungswerte geführt hat. Gerade das Zulassen von Gesprächspausen stellt sich vielfach als Hürde für Interviewer dar. Insofern hat es mir mein beruflicher Hintergrund ermöglicht, dass Interviewpartner mit ihren Erfahrungshorizonten in vertrauensvoller Gesprächsatmosphäre im Mittelpunkt stehen konnten. 


\section{Präkonzepte}

Das von Glaser (2012) postulierte Diktum, Präkonzepten jedweder Art in der qualitativen Forschung keinen Raum zu geben, sondern stattdessen den Anspruch „empty head versus open mind“ zu beherzigen, ist erkenntnistheoretisch weder nachvollziehbar noch vertretbar, da ein Forscher per se keinen „leeren Kopf“ haben kann. Prägung ist immer gegeben, Vorannahmen müssen demzufolge vorhanden sein. Wird Glasers Diktum jedoch nicht erkenntnistheoretisch betrachtet, sondern psychologisch, gewinnt es insofern an Bedeutung, als qualitative Sozialforscher demnach immer die Grundhaltung zu beherzigen haben, sich ihrer Präkonzepte bewusst zu sein, sie gedanklich einzufangen und mit diesen sensibel in Selbstdistanz umzugehen. Diese Idee einer Dezentrierung entspricht dem selbstreflexiven Einnehmen einer Metaebene, ganz im Verständnis der RGT (vgl. Breuer et al., 2017, S. 111 f.). Dabei ist zu berücksichtigen, dass sich weitere Sinnkonstruktionen im Forschungsprozess entwickeln, erweitern und verändern sowie Einfluss auf den Erkenntnisgewinn nehmen können. Daher stellt bei der RGT das Be- und Überdenken von Annahmen eine Grundhaltung während der gesamten Methodenanwendung dar. Der methodenkritischen Reflexion ist dabei ein breiter Raum zu geben. Meine Präkonzepte reflektierte ich eigenständig sowie in Kodier-Arbeitsgruppentreffen und in Supervisionssitzungen mit den im elektronischen Zusatzmaterial in Anhang G wiedergegebenen Fragestellungen nach Breuer (2018) zur „kontinuierlichen Verortung als Forschersubjekt".

\section{Postskripta}

$\mathrm{Zu}$ jedem der 35 Interviews habe ich die Informationen, die mir unmittelbar vor und nach dem Ausschalten des Aufnahmegeräts zugetragen worden sind, sowie unterschiedlichste Beobachtungen und Besonderheiten gewissenhaft in Postskripta dokumentiert. Dafür nutzte ich für jedes Interview einen mehrstündigen Zeitslot unmittelbar nach Gesprächsende und habe bei jeder Ausarbeitung eine selbst entwickelte, einheitliche Gliederungsstruktur für alle Postskripta befolgt. Ebenso habe ich vorab erhaltene und recherchierte Informationen sowie Angaben zur Koordination der Interviewtermine notiert. Jede damit verbundene Nachbereitung diente mir zur Planung und Vorbereitung des Folgeinterviews, aber auch zur Rückspiegelung auf vorherige Interviews. Ebenso habe ich Störungen im Gesprächsverlauf dokumentiert. Beispielsweise fielen mir selbst missglückte Fragestellungen z. B. mit Suggestivcharakter auf, in einer anderen Situation auch gefühlte Antipathie gegenüber einer Aussage eines IP oder z. B. visuelle Beobachtungen, die mich ablenkten. Bei der späteren Datenanalyse konnte ich auf diese Weise eine dadurch 
eventuell bedingte eingeschränkte Aussagekraft für den Auswertungsprozess einbeziehen und zudem meinen eigenen, personenbezogenen Lernprozess in der Rolle als Interviewer für die Planung von Folgeinterviews nutzen.

Sichergestellt habe ich darüber hinaus für jedes Interview, das komplette Gespräch am Tag der Datenerhebung und ansonsten am Folgetag noch einmal anzuhören und meine Notizen zu komplettieren. Hierbei fertigte ich ebenfalls Memos an, die ich später in die softwaregestützt eingearbeitete Transkription übertragen habe.

\section{Fallauswahl, Fallkontrastierung und Erhebungszeitraum}

Die theoretisch geplante Fallauswahl im Bottom-up-Ansatz und bei nachvollziehbar begründeter Fallkontrastierung erfordert eine gewissenhafte Recherche hinsichtlich der Eignung eines jeden Interviewpartners zu den Kriterien, denen eine Relevanz für das Beantworten der zentralen Forschungsfrage zugeschrieben wird. Diese Weiterentwicklung dokumentierte ich für jedes Interview in den Postskripta, einhergehend mit der Entwicklung meiner Sampling-Matrix. Zudem konnten die Kodier-Arbeitsgruppen zur Reflexion von bestmöglich infrage kommenden Folgeinterviewpartnern genutzt werden, also zur Gestaltung der minimalen und maximalen Fallkontrastierung.

Bereits aus der begrenzten Anzahl geeigneter Interviewpartner im Forschungsfeld ergibt sich mein Anspruch an ein professionelles Auftreten von Anfang an. Denn Qualität und Verlauf meiner Ansprache können bei angefragten Social Entrepreneuren richtungsweisend sein für deren Bereitschaft zur Teilnahme an einem Interview. Daher habe ich vorbereitend für die verschiedenen Interviewpartner versucht herauszuarbeiten, was deren Motivation sein kann bzw. worin sie einen Nutzen erkennen können, sich für ein Interview zur Verfügung zu stellen. Diese Vorüberlegungen habe ich bei meiner persönlichen E-Mail-Ansprache argumentativ eingebaut. Bei meiner stets wie angekündigt erfolgten telefonischen Kontaktaufnahme konnte ich dies meist aufgreifen und oft auch als Gesprächsaufhänger nutzen. Mehrfach gaben mir angefragte Social Entrepreneure die Rückmeldung, dass sie Einladungen zu Befragungen i. d. R. ablehnen, ich jedoch mit meiner Argumentation überzeugend aufgetreten sei. Insbesondere die Zusicherung, ein Exemplar der Studienveröffentlichung zu erhalten, überzeugte. Mehrere IP betonten und bedauerten zugleich, dass sie in der Vergangenheit an Befragungen teilgenommen hatten, ohne jemals die daraus hervorgegangenen Ergebnisse übermittelt bekommen zu haben.

Einerseits ist es mir beim theoretischen Sampling - trotz mehrerer Interviewabsagen und unbeantwortet gebliebener Anfragen - für alle Entwicklungsschritte hinreichend gelungen, geeignete Interviewpartner zu recherchieren und für ein Gespräch zu gewinnen. Andererseits sorgten seitens der Interviewpartner späte 
Rückmeldungen, mehrere Terminverschiebungen, bis zu mehrmonatige Wartezeiten und in einem Fall eine vergessene Verabredung zu einer anderen Reihenfolge als geplant. Mit meiner Sampling-Matrix und dem strukturierten Gesamtüberblick an Informationen, die ich in den Postskripta festgehalten habe, konnte ich dennoch überprüfen und feststellen, dass die Erhebungsqualität dadurch nicht beeinträchtigt worden ist. Dabei konnte ich sogar sicherstellen, dass der Befragungsprozess so lange erfolgte, bis kein neuer Erkenntnisgewinn mehr generiert werden konnte und auch nicht mehr zu erwarten war. Wie bereits angedeutet, zeichnete sich die theoretische Sättigung mit dem 34. Interview ab. Sicherheitshalber habe ich ein 35. Interview, dessen Wartezeit bis zum Termin drei Monate betragen hat, durchgeführt und ausgewertet, aus dem tatsächlich kein bislang unentdeckter Erkenntnisgewinn hervorgegangen ist. Dennoch kann ich trotz gewissenhaft ausgewählten Fällen und eingehaltener komparativer Datenanalyse keineswegs ausschließen ,schwarze Schwäne" übersehen zu haben: Nicht auszuschließen ist die Existenz weiterer Fälle mit weiteren Merkmalsausprägungen, die in dieser Untersuchung unentdeckt geblieben sind. Zudem ist in jedem Fall ein willkürlicher Abbruch in der komparativen Analyse vorzunehmen. Das Entscheiden für und gegen theoretische Überlegungen passiert letzten Endes willkürlich, denn ,,[d]ie Möglichkeiten zu vergleichen sind im Prinzip unbegrenzt, und deshalb müssen die Gruppen nach theoretischen Kriterien ausgesucht werden“ (Glaser \& Strauss, 1998, S. 55). Dies limitiert die Aussagekraft meiner Untersuchung dementsprechend.

Zwar konnte ich sämtliche identifizierten Einflussbedingungen bei mehreren Fällen intensiv reflektieren. So konnte ich für alle identifizierten Kriterien sicherstellen, diese bei maximaler Ähnlichkeit (z. B. Scheitern bis zur Insolvenz), also minimal kontrastiert, untersucht zu haben. Allerdings konnte ich aufgrund der Unterschiedlichkeit aller Interviewpartner die gegebene Kriterienvielfalt innerhalb eines Falls nicht für mehrere Fälle minimal kontrastieren.

Beim Theoretical Sampling entstand kein Zeitdruck. Zwar konnte ich erst Ende März 2018 mit der ersten Datenerhebung beginnen, also mit fast zweimonatiger Verspätung infolge mehrerer Terminverschiebungen durch IP1. Beim Promotionsstart habe ich das Beenden der Datenerhebung kalkuliert auf Herbst 2019 und das letzte Interview fand im September 2019 statt, also innerhalb des Planungshorizonts. Während der anderthalb Jahre dazwischen konnte ich kontinuierlich Interviews führen. Einen halbjährlichen Zeitpuffer für die Datenerhebung, den ich mir vorsichtshalber gesetzt hatte, musste ich nicht in Anspruch nehmen.

\section{Leitfadengestaltung}

Wie in Abschnitt 3.2.2 dargestellt, habe ich bei der Leitfadengestaltung auf das Einhalten des Prinzips der Offenheit und der Flexibilität geachtet. Die inhaltlich 
strukturierte stichwortartige Gestaltung schützt vor dem Ablesen und ermöglichte es mir gut, Fragen aus der Situation heraus zu formulieren und mich in meiner Ausdrucksweise meinen Gesprächspartnern anzupassen. Der Prozesscharakter wird auch mit der variierten Interviewdauer deutlich. Die Transkriptionen und deren zugehörige Postskripta zeigen, wie ich Raum für eine wertschätzende Erzählaufforderung in einer alltagsnahen und vertrauensförderlichen Gesprächssituation geschaffen habe, bei der die Handlungs- und Deutungsmuster der Social Entrepreneure im Gesprächsmittelpunkt stehen konnten.

In der Vorbereitung auf die Ausarbeitung der Leitfäden habe ich mich in Arbeitsgruppentreffen und in Supervisionssitzungen mit meinen apriorischen Strukturen und präkonzeptuellen Annahmen auseinandergesetzt, verbunden mit der Zielsetzung, bestmöglich sicherzustellen, dass ich in die Ausführungen der IP keine mentalen Riegel schiebe, die das Emergieren von Daten unterbinden oder einseitig zu sehr fokussieren könnten. Dabei erschien es mir bereits in der Vorbereitung auf mein Probeinterview, das ich rechtzeitig vor dem eigentlichen Erhebungsbeginn geführt habe, sinnvoll, das problemzentrierte Interview mit narrativen Anteilen zu kombinieren, um im Forschungsverlauf ein Thema zunehmend fokussieren zu können und in dieser Phase erst entscheiden zu dürfen, welche Elemente wichtig und welche weniger wichtig sind. Beispielsweise ermöglichte es mir das Integrieren der narrativen Anteile, die weiter oben skizzierte Einstiegs- und Abschlussfrage zu stellen und mich gemeinsam mit meinem Interviewpartner dazu zu verständigen. Ebenso verschafften sie mir einen Spielraum für das Stellen von Verständnisfragen und dem Nachfragen zum Vertiefen eines Themas, was der Validität meiner Ausarbeitung zugutekommen kann.

\section{Kontaktgestaltung im Interview, Gesprächsführung und kommunikatives Validieren}

Zwangsläufig wirkt das Forschungsfeld auf den qualitativen Forscher ein, so dass er nicht nur agiert, sondern auch auf Eindrücke aus dem Forschungsfeld, Berichte und die Persönlichkeit der Interviewten reagiert. Zur (Selbst-)Reflexion zog ich vor allem meine Postskripta-Notizen aus den Spalten „Kontaktaufnahme“, „Kontaktgestaltung zwischen Interviewer und Interviewtem inkl. Gedanken, Verhalten und Gefühle des Interviewers“, ,,Gesprächssetting“, „Interviewsituation, Interviewverlauf“, „Hervorzuhebendes Verhalten der interviewten Person und Wirkung auf den Interviewer“, „Allgemeine Beobachtungen und persönlicher Kontakt nach Ausschalten der Aufnahmegeräte“ und „Allgemeine Anmerkungen“ heran und reflektierte diese sowohl alleine als auch im Rahmen der Supervisionssitzungen und in Arbeitsgruppentreffen. Dies half mir, mich von meinem Forschungsfeld zu 
dezentrieren und meine subjektiven Beobachtungen sowie die in mir als Forscherperson begründeten Störungen für ein Reflektieren von forschungsfragenrelevanten Kriterien zu nutzen, was im Sinne der RGT ,innere Zensuren“ reduziert, der Erforschung subjektiver Relevanzsysteme somit dienlich ist und bei der Konzeptentwicklung erkenntnisproduktiv wirken kann (vgl. Breuer et al., 2019, S. 10, 85, 171). Zwar lässt sich dieser interaktive Gestaltungsspielraum nicht standardisieren. Dennoch habe ich mit meiner transparenten Verfahrensdokumentation, die bei der softwaregestützten Datenverarbeitung das umfangreiche Ausformulieren von Memos beinhaltet und ebenfalls die in meinen Kodier-Arbeitsgruppen entstandenen Kommentierungen protokollarisch festhält, eine intersubjektiv nachvollziehbare Vergleichbarkeit sicherstellen können (ebd., S. 272 ff.). Auf diese Weise konnte ich meinen methodologisch roten Faden sichtbar machen.

Wie bereits angedeutet, erfordert die Kontaktgestaltung eine kompetente Gesprächsführung. Das Reflektieren meiner kommunikativen Kompetenz nahm ich bereits gesprächsvorbereitend vor, indem ich mir zu meinen Stichwörtern auf den Leitfäden beispielhaft Fragen ausformulierte und dabei übte, ungeeignete Fragestellungen (z. B. geschlossene W-Fragen und Suggestivfragen) zugunsten offener Fragestellungen (beispielsweise durch die eine Frage einleitende Formulierung mit „Inwieweit") zu vermeiden und mir dies zu vergegenwärtigen.

Sowohl mit den 35 Interviewpartnern als auch mit den fünf Fachgesprächspartnern fand ein zum Teil intensiver und aufschlussreicher Nachkontakt statt: telefonisch und per E-Mail. Diese Kommunikation diente mir dazu, erst im Zuge der Datenauswertung entstandene Unklarheiten aus dem Weg zu räumen sowie ggf. Eindeutigkeit zu erlangen, wenn erst beim Auswerten ein Interpretationsspielraum im Verständnis des Gesagten entstanden ist. Den dabei jeweils generierten Erkenntnisgewinn habe ich als Memos direkt bei der softwaregestützten Datenauswertung festgehalten und weiterverarbeitet. Die verständnisgenerierende Rückkopplung stellt somit einen bedeutsamen Anteil der kommunikativen Validierung dar und ermöglicht mir zudem darauf zu achten, dass Erkenntnisse nicht auf meiner willkürlichen Interpretation basieren, sondern aus den Erhebungsdaten direkt gewonnen werden können, was der ökologischen Validität zugutekommt.

Mit diesen Ausführungen ist deutlich geworden, dass Objektivität als klassisches zentrales Gütekriterium der quantitativen Forschung, bei der auch die Datenerhebung weder vom Forscher noch von Befragten subjektiv geprägt sein darf, nicht zur Bewertung der Güte einer RGT-Studie herangezogen werden kann - und das ist auch gut so (vgl. z. B. Kruse, 2014, S. 55; Breuer et al., 2019, S. 399 ff.; Steinke, 2013, S. 323 ff.). 


\section{Datenquellen-Triangulation}

$\mathrm{Zu}$ mehreren IP konnte ich neben deren Homepages weitere forschungsfragenrelevante Dokumente, auch Audio- und Videodateien, eruieren. Darüber hinaus überreichten mir fünf IP und zwei Fachgesprächspartner im Rahmen der Gespräche Bücher und Fachartikel, die ihre Argumentationen untermauern. Ebenso zählen die Postskripta dazu. Diese Dokumente habe ich bei der Datenauswertung auszugsweise unterstützend berücksichtigt. Allerdings habe ich die herangezogenen Audio- und Videodateien weder transkribiert noch nach einer Systematik vollständig ausgewertet.

Nach der Darstellung und methodenkritischen Reflexion der Datenerhebung folgt nun die Beschreibung der standardisierten wie auch strukturierten Aufbereitung der Daten als Vorbereitung für das Analysieren und Auswerten der Interviews.

\subsection{Datenaufbereitung und softwaregestützte Datenverarbeitung}

Die elektronisch aufgezeichneten Interviews sind in Schriftform zu transferieren und darüber hinaus handhabbar aufzubereiten. Neben dem Befolgen eines Regelwerks für das Transkribieren der Daten sind auch Grundlagen für das weitere Arbeiten mit den Daten zu schaffen, die das Transparenzgebot bei der Datenauswertung gewährleisten.

\subsubsection{Transkriptionsregeln und Transkriptionsprozess}

Mit dem Transkribieren sämtlicher elektronisch aufgezeichneten Daten wurde ein professionelles, in der Transkription qualitativer Daten langjährig erfahrenes Schreibbüro beauftragt. Anhang $\mathrm{H}$ im elektronischen Zusatzmaterial zeigt die angewandten Transkriptionsregeln.

Alle Interviews mit SE werden während der Transkription regelgeleitet anonymisiert, wobei darüber hinaus im Rahmen der Ergebnisdarstellung und Diskussion die in Endnoten dargestellten Zitate in erweiterter Form anonymisiert werden, sofern dies notwendig erscheint. Ebenso wird vereinbarungsgemäß eines der fünf Fachgespräche anonymisiert. Wie bereits erwähnt, zeigen die anderen vier Fachgesprächspartner sich damit einverstanden, dass ihre Gespräche ohne Anonymisierung verarbeitet werden dürfen. 
Obwohl mehrere SE von sich aus abschließend anmerken, auf eine Anonymisierung ihres Interviews zu verzichten, wird dennoch an den meisten Stellen anonymisiert. Allerdings werden zur Illustration vereinzelte Zitate herangezogen, die für das Beantworten der zentralen Forschungsfrage eindrucksvoll erscheinen und einen näheren Einblick in Arbeitsanforderungen verschaffen, was die Wahrscheinlichkeit eines personenbezogenen Rückschlusses entsprechend erhöht.

Da sich beim Kombinieren von Interviewzitaten die Anonymität der Befragten eventuell nicht mehr aufrechterhalten lässt, sondern Fakten damit leichter zuordnen lassen könnten, werden den Interviewpartnern neben dem IP-Zahlenkürzel (von 1 für IP1 bis 35 für IP35) weitere Pseudonyme zugewiesen. Deren systematische Zuordnung kann für den Leser nicht erkennbar sein. Hierfür werden griechische Kleinbuchstaben (z. B. IP $\alpha$, IP $\beta$, IP $\gamma$ ), römische Zahlen (z. B. IPI, IPII, IPIII) und deutsche Groß- und Kleinbuchstaben (z. B. IPA, IPB, IPC; IPa, $\mathrm{IPb}, \mathrm{IPc})$ herangezogen. Da für einen Beleg nicht Zitate von allen 35 IP herangezogen werden, stellt die begrenzte Auswahl an Buchstaben eines Alphabets keine Limitierung für die Zitation dar. In der späteren Datenauswertung wird diese Vorgehensweise zur Pseudonymisierung bedarfsorientiert abermals angewandt, wenn eine Kombination an Zitaten einen Hinweis auf die Identität eines IP liefern könnte und dies bei der erstmaligen Pseudonymisierung noch nicht erkannt wurde.

\subsubsection{Softwaregestützte Datenverarbeitung: MAXQDA}

Während des Datenauswertungsprozesses wurde unterstützend mit der Datenverarbeitungssoftware MAXQDA gearbeitet: zu Studienbeginn mit der Version 2018 und ab Februar 2020 mit dem Update 2020. Anhang B im elektronischen Zusatzmaterial beinhaltet die Auflistung der absolvierten SoftwareAnwenderschulungen. Allerdings wurden bewusst nur solche Funktionen genutzt, die sich für die gegebene qualitative Datenauswertungssituation als zielführend erwiesen haben:

Sämtliche Transkriptionen wurden eingespeist; sie konnten trotz ihrer Komplexität ohne Verlust eines Überblicks miteinander verglichen sowie bedarfsorientiert synchron analysiert werden. Die schnelle, passgenaue Eingabe von sowie Suche nach Memos und Kodes wurde damit ebenso gesichert wie die zuverlässige und effiziente Recherche zu genutzten Begriffen und Aussagen der Gesprächspartner; dies sowohl innerhalb eines Interviews als auch im Abgleich zwischen den verschiedenen Interviews. Dabei ließen sich beispielsweise unterschiedliche 
„Experimente“ des Kodierens und Versuche des Kategorisierens parallel abspeichern und im Vergleich bewerten, ohne den Überblick sowie den roten Faden zur Zielsetzung zu verlieren. Ebenso ließ sich anschaulich abbilden und schnell herausarbeiten, in welchen Interviews an welchen Stellen bestimmte Inhalte vertieft bzw. Fragestellungen thematisiert wurden.

Darüber hinaus ist mit der Anwendung von MAXQDA vereinbar, unterschiedliche Auswertungsverfahren parallel anzuwenden. Diese Option wurde in Anspruch genommen, da neben der Auswertung nach der RGT zwei qualitativ-inhaltsanalytische sowie ein metaphernanalytisches Verfahren zum Einsatz kamen.

Weitere Software-Funktionen kamen nicht bzw. nur marginal zum Einsatz.

\subsubsection{Methodenkritische Reflexion zur Datenaufbereitung}

Mit der Beauftragung des professionellen Schreibbüros konnte ich gewährleisten, dass sämtliche elektronischen Aufzeichnungen unter Wahrung des Datenschutzes, sehr zeitnah und gewissenhaft nach allen vorgegebenen Transkriptionsregeln verarbeitet wurden. Zudem konnte ich für alle Aufzeichnungen das Vier-Augen- und Vier-Ohren-Prinzip einhalten, demgemäß das Korrekturlesen von einer anderen als von der transkribierenden Person durchgeführt wird, wodurch sich eingeschlichene Transkriptionsfehler optimal erkennen und beseitigen lassen. Da ich das Interview führte, kann nur ich mich an die Erhebungssituation erinnern und beispielsweise Hintergrundgeräusche in einen interpretationsbefreiten Sinnzusammenhang bringen, so dass ich entsprechende Textstellen korrekt komplettieren konnte. Beim Korrekturlesen habe ich weitere Memos zu Emotionen, zur Prosodie und zum Hintergrundgeschehen angefertigt und auch erste Kodes generiert, die ich abschließend in die Datenverarbeitungssoftware MAXQDA übertragen habe, nachdem ich die Korrektur gelesene Transkription dorthin übertragen habe.

Vor dem Übertragen der Textdateien in die MAXQDA-Software habe ich die gesamten Interviews noch einmal auf ihre Anonymisierung überprüft. Zur Wahrung der Anonymität befinden sich die Postskripta und die vollständigen Transkriptionstexte nicht in dieser Arbeit. Sie wurden, ebenso wie alle weiteren Forschungsprozess-Dokumentationen, im Rahmen der Begutachtung in einem separaten, passwortgeschützten digitalen Datenträger uneingeschränkt zur Verfügung gestellt.

Da einer der befragten SE sehr gebrochenes Deutsch spricht, habe ich nachträglich seine Ausdrucksweise zur Wahrung seiner Anonymität stark angepasst, ohne dabei die inhaltliche Aussage zu verändern. Dennoch muss ich mir ob der 
gegebenen Sprachmängel darüber im Klaren sein, dass dieser Interviewpartner in seiner Wortwahl sehr eingeschränkt war und dadurch eventuell nicht immer das zum Ausdruck bringen konnte, was er zum Ausdruck bringen wollte. Zur bestmöglichen Sicherung des vom IP beabsichtigten Textverständnisses wandte ich während dieses Interviews besonders intensiv die Gesprächsführungstechnik Paraphrasieren an.

Kein Anonymisierungsverfahren kann hundertprozentige Sicherheit vor Rekonstruktionen bieten. Auch die zuvor geschilderte Verfahrensweise zur Pseudonymisierung von IP-Zitaten löst das Spannungsfeld nicht sicher auf, dass Kontextwissen von Lesern zu Rückschlüssen auf den SE führen kann. Um dem bestmöglich entgegenzuwirken, habe ich personengebundene und institutionelle Daten bestmöglich abstrahiert und nicht jede geeignete Textstelle als Belegquelle angegeben, sondern Zitationen bedarfsorientiert vereinzelt vorgenommen.

Bis hier wurde der Weg geebnet für eine solide, empirisch abgesicherte Datenauswertung, die nachfolgend ausführlich dargestellt und bewertet wird.

\subsection{Datenauswertung}

Sämtliche Interviewtranskriptionen wurden nach einer komplexen RGTKodiermethodik ausgewertet, deren Basis das Kodierparadigma von Strauss (1991) bzw. von Strauss und Corbin (1996) darstellt. Punktuell wurde ergänzend metaphernanalytisch ausgewertet. Die Fusion dieser beiden Verfahren stellt eine legitime „eklektische Methodenkonfiguration“ (Breuer et al., 2017, S. 359) dar im Rahmen der „Diversifikation der Grounded Theory-Wege“ (ebd., S. 24).

Darüber hinaus wurden die Interviewtranskriptionen zu Teilfragestellungen qualitativ inhaltsanalytisch ausgewertet; dies nicht im Sinne einer symbiotisch wirksamen Methoden-Triangulation und somit nicht im Kontext der RGTKodiermethodik, sondern als parallel angewandte, eigenständig zu betrachtende Datenauswertung.

Alle angewandten Auswertungsverfahren werden nun der Reihe nach besprochen. Mit der Kurzübersicht in Tabelle 3.1 wird der dieser Arbeit zugrunde liegende Bedeutungsgehalt von Fachtermini wiedergegeben. Diese Begriffe finden vorwiegend, aber nicht nur bei Grounded-Theory-Ansätzen Anwendung. Bei der Durchsicht der Fachliteratur zu qualitativen Methoden zeigt sich, dass die Bedeutung der darin verwendeten Begriffe meist nicht präzisiert wird: Die Begriffe werden entweder nicht genauer definiert oder uneinheitlich verwendet (vgl. z. B. Mayring, 2016; Flick, Kardorff \& Steinke, 2013; Lamnek, 2010; vgl. auch einen Systematisierungsversuch von Kruse, 2014, S. 387 ff.). 
Tabelle 3.1 Kurzdefinitionen zur angewandten Grounded-Theory-Terminologie. (eigene Darstellung)

\begin{tabular}{|c|c|c|}
\hline Begriff & Kurzdefinition & Zugehörige Tätigkeit mit Zielsetzung \\
\hline $\begin{array}{l}\text { Kode } \\
\text { (auch: Code) }\end{array}$ & $\begin{array}{l}\text { Im Datenmaterial } \\
\text { identifizierte und } \\
\text { daraufhin textlich } \\
\text { aufbereitete Aussage mit } \\
\text { einem für die } \\
\text { Forschungsfrage } \\
\text { relevanten } \\
\text { Bedeutungsgehalt. }\end{array}$ & $\begin{array}{l}\text { Kodieren: Einer transkribierten Textstelle } \\
\text { wird ein höherer Abstraktionsgrad } \\
\text { zugeordnet. Dabei können dem Kode } \\
\text { weitere transkribierte Textstellen mit hierzu } \\
\text { passendem Bedeutungsgehalt zugeordnet } \\
\text { werden. Kodes werden beim Kodieren } \\
\text { permanent miteinander verglichen und } \\
\text { überarbeitet, ergänzt und ggf. wieder } \\
\text { verworfen. } \\
\text { Nach Strauss (und Corbin) wird } \\
\text { unterschieden zwischen offenem, axialem } \\
\text { und selektivem Kodieren (siehe Erläuterung } \\
\text { zum Kodierparadigma in Abschn. 3.4.1). }\end{array}$ \\
\hline $\begin{array}{l}\text { Konzept (auch: } \\
\text { Concept) }\end{array}$ & $\begin{array}{l}\text { Gruppe an Kodes, die in } \\
\text { ihrer Zusammenschau } \\
\text { einen gemeinsamen } \\
\text { Bedeutungsgehalt/eine } \\
\text { zusammenhängende } \\
\text { Aussage aufweisen. }\end{array}$ & $\begin{array}{l}\text { In der GT wird das „Konzeptualisieren“ } \\
\text { oftmals beim „Kategorisieren“ subsumiert, } \\
\text { was auch der Vorgehensweise in dieser } \\
\text { Studie entspricht. Siehe daher in der } \\
\text { übernächsten Spalte zu „Kategorisieren“ bei } \\
\text { „Kategorie“. }\end{array}$ \\
\hline Indikator & $\begin{array}{l}\text { Konkretes Textelement in } \\
\text { der Transkription, das als } \\
\text { (weiterer) Hinweis auf die } \\
\text { Existenz eines Konzepts } \\
\text { bzw. einer Kategorie zu } \\
\text { verstehen ist. Der } \\
\text { Prozessschritt „,vom Kode } \\
\text { zum Konzept“" bzw. ,vom } \\
\text { Kode zur Kategorie“ kann } \\
\text { erst über Indikatoren } \\
\text { sichtbar und bewertbar } \\
\text { werden. }\end{array}$ & $\begin{array}{l}\text { Das Arbeiten mit einem } \\
\text { Konzept-Indikator-Modell (ursprünglich } \\
\text { entwickelt von Glaser und Strauss), wobei } \\
\text { sämtliche Indikatoren untereinander auf } \\
\text { ihre Sinnkonsistenz verglichen werden. } \\
\text { Nimmt die Anzahl an sinnkonsistenten } \\
\text { Indikatoren in einer Gruppe zu, steigt damit } \\
\text { der Sättigungsgrad der Eigenschaften für } \\
\text { das entsprechende Konzept bzw. die } \\
\text { entsprechende Kategorie. } \\
\text { Für das Herausarbeiten von Indikatoren } \\
\text { können unterschiedliche } \\
\text { Auswertungsmethoden mit ihren } \\
\text { zugehörigen Auswertungstechniken } \\
\text { angewandt werden. }\end{array}$ \\
\hline
\end{tabular}


Tabelle 3.1 (Fortsetzung)

\begin{tabular}{|c|c|c|}
\hline Begriff & Kurzdefinition & Zugehörige Tätigkeit mit Zielsetzung \\
\hline Kategorie & $\begin{array}{l}\text { Mehrere Konzepte, die in } \\
\text { ihrer Gruppierung eine } \\
\text { übergeordnete } \\
\text { Sinnstruktur und eine } \\
\text { Basis für die spätere } \\
\text { Theoriebildung darstellen. }\end{array}$ & $\begin{array}{l}\text { Kategorisieren: Identifizierte Konzepte } \\
\text { werden untereinander auf } \\
\text { Beziehungsverhältnisse geprüft und im } \\
\text { positiven Fall als zusammenhängende } \\
\text { Konzepte dargestellt. }\end{array}$ \\
\hline $\begin{array}{l}\text { Schlüssel-/ } \\
\text { Kernkategorie }\end{array}$ & $\begin{array}{l}\text { Die zentral bedeutsame } \\
\text { Kategorie, mit der eine } \\
\text { Theorie stabil gestützt } \\
\text { wird. }\end{array}$ & $\begin{array}{l}\text { Schlüssel- bzw. Kernkategorisieren: } \\
\text { Sämtliche gebildete Kategorien werden in } \\
\text { eine einzige Lagebeziehung zueinander } \\
\text { gebracht und nicht nur ,hierarchisch } \\
\text { geordnet“, sondern ,priorisiert“, wobei die } \\
\text { Schlüssel- bzw. Kernkategorie sich durch } \\
\text { einen zentral bedeutsamen Stellenwert von } \\
\text { allen anderen Kategorien abgrenzt. }\end{array}$ \\
\hline Subkategorie & $\begin{array}{l}\text { Die Konzepte einer } \\
\text { Kategorie lassen sich } \\
\text { wiederum klassifizieren, } \\
\text { da sie in } \\
\text { Sinnzusammenhängen } \\
\text { zueinanderstehen. }\end{array}$ & $\begin{array}{l}\text { Subkategorisieren: Identifizierte } \\
\text { Subkategorien werden in ihrer Gesamtheit } \\
\text { ins Beziehungsgefüge zueinander gebracht } \\
\text { und in einer Systematik abgebildet. Es gibt } \\
\text { Systematisierungsalternativen (z. B. } \\
\text { Typenmodell, Handlungsmodell, } \\
\text { Prozessmodell). Subkategorisiert wird u. a. } \\
\text { beim axialen Kodieren (siehe separate } \\
\text { Erläuterung). }\end{array}$ \\
\hline Phänomen & $\begin{array}{l}\text { In der Praxis sich } \\
\text { widerspiegelnde } \\
\text { Kategorie. Der Kern eines } \\
\text { Problems wird in der } \\
\text { Praxis als Phänomen } \\
\text { sichtbar. }\end{array}$ & - \\
\hline Dimension & $\begin{array}{l}\text { Spezifische Ausprägung } \\
\text { einer fokussierten } \\
\text { Eigenschaft, oftmals als } \\
\text { Skalierung dargestellt } \\
\text { (z. B. „,sehr zugänglich“ } \\
\text { vs. ,gar nicht } \\
\text { zugänglich“). }\end{array}$ & $\begin{array}{l}\text { Dimensionalisieren: Vorgang des } \\
\text { Bewertens einer Eigenschaft, erfolgt } \\
\text { oftmals innerhalb eines Kontinuums bipolar } \\
\text { skaliert. }\end{array}$ \\
\hline
\end{tabular}


Tabelle 3.1 (Fortsetzung)

\begin{tabular}{|c|c|c|}
\hline Begriff & Kurzdefinition & Zugehörige Tätigkeit mit Zielsetzung \\
\hline Theorie & $\begin{array}{l}\text { In sich schlüssige } \\
\text { Gruppierung aller } \\
\text { Kategorien zu einem } \\
\text { übergeordneten Ganzen. } \\
\text { Das Gesamtgebilde hierzu } \\
\text { stellt ein Muster dar, das } \\
\text { die zentrale } \\
\text { Forschungsfrage } \\
\text { beantwortet und sich auf } \\
\text { andere Fälle anwenden } \\
\text { lässt. }\end{array}$ & $\begin{array}{l}\text { Theoriebildung: Das finale, übergeordnete } \\
\text { Herausarbeiten von Erkenntnisgewinn, aus } \\
\text { dem sich ein „regelgeleiteter Charakter“ } \\
\text { ableiten lässt, der auf andere Fälle } \\
\text { angewandt werden kann bzw. an anderen } \\
\text { Fällen gespiegelt werden kann. } \\
\text { Neben Theorien können vor allem auch } \\
\text { Modelle und Typologien gebildet werden. }\end{array}$ \\
\hline
\end{tabular}

\subsubsection{Datenauswertung nach der Reflexiven Grounded Theory (RGT)}

Für die Datenauswertung nach der RGT wurde jedes Interview zu Beginn mindestens ein weiteres Mal vollständig und darüber hinaus bedarfsorientiert auszugsweise angehört, wobei weitere Memos und Kodes generiert wurden. Das bedarfsorientierte Zurückgreifen auf die Audiodateien während der gesamten Auswertungsphase ermöglichte ein Vergegenwärtigen der Erhebungssituation.

Die Datenauswertung nach der RGT fand sowohl eigenständig als auch in den bereits benannten Kodier-Arbeitsgruppen statt (vgl. Anhang B im elektronischen Zusatzmaterial). An die parallel zum anderthalbjährigen Erhebungsprozess vollzogene Datenauswertung schloss sich ein einjähriger, komplexer Auswertungsprozess an. Dabei wurde sichergestellt, dass beim Generieren des neu- und höherwertigen Sachverstands, dessen Verstehensprozess sich vom subjektiven Alltagsverständnis abzugrenzen hat, Vorverständnis und Textverständnis ebenso ineinandergreifen (hermeneutischer Zirkel I) wie Textteil und Textganzes (hermeneutischer Zirkel II). Zur Beantwortung der zentralen Forschungsfrage konnten aus dem Datenmaterial letztendlich deutlich über 1.000 Kodes herausgearbeitet werden. Einige dieser Kodes besitzen in mehrerlei Hinsicht eine Aussagekraft, was dementsprechend aufbereitet wurde und Mehrfachkategorisierungen einforderte. Sämtliche Kodes lassen sich systematisch zueinander in Verbindung stehend kategorisieren. Während des Auswertungsprozesses war die Kode-Anzahl zuweilen weitaus höher, wobei das Überarbeiten und Verwerfen vieler Kodes zur deutlichen Reduktion geführt hat. Denn die Zusammenhänge zwischen den erhobenen Informationen ergeben sich erst durch den ständigen reflexiven Bezug im 
Kontext der Datenauswertungsmethode. Auf diese Weise kann sich eine gesättigte Theorie aus dem Material heraus entwickeln (vgl. Breuer et al., 2017, S. 417 ff.). Demzufolge ist die „Grounded-Theory (...) keine Theorie, sondern eine Methodologie, um in den Daten schlummernde Theorien zu entdecken" (Legewie \& Schervier-Legewie, 1995, S. 70 f.).

Nach Strauss (1991) bzw. Strauss und Corbin (1996) erfolgt der Kodierprozess in der Zusammenschau als sog. Kodierparadigma, bestehend aus drei aufeinanderfolgenden Etappen: dem offenen, dem axialen und dem selektiven Kodieren.

\section{Offenes Kodieren}

Beim offenen Kodieren wird das Datenmaterial ,,aufgebrochen“: Die darin enthaltenen Sinneinheiten werden herausgearbeitet und begrifflich aufbereitet (vgl. Strauss \& Corbin, 1996, S. 45 ff.). Breuer (2009) versteht dies als „,eine Art assoziatives Brainstorming zu möglichen Bedeutungen (...) mit dem Ziel der Bildung von typisierenden Sprachausdrücken, von Begriffen höheren Allgemeinheitsgrades" (ebd., S. 80). Zur Verfahrensweise eignen sich vor allem W-Fragen (vgl. Böhm, 2013, S. 477 f.), die an den gesamten Transkriptionstext immer wieder herangetragen werden: beginnend innerhalb eines Interviews in der ersten Zeile und innerhalb kleinerer Absätze, sodann auch in längeren Passagen und dies bis zum Ende der Transkription, dann auch rückblickend innerhalb einer Transkription sowie schließlich interviewübergreifend. Die Funktion dieses „,Sequenzialitäts-Prinzips“erläutern Breuer et al. (2017) wie folgt: ,Wenn ich , das Ende“ bereits kenne, ist mir der gedankliche Zugriff auf Fragen und Unsicherheiten versperrt oder erschwert, die mich zuvor (ohne dieses Wissen) bewegen bzw. beschäftigen könnten. Mein gedanklicher Such- und Assoziationsraum wird so verkleinert, meine datenbezogenen Phantasien werden eingeschränkt - und das kann von Nachteil sein für das Zustandekommen neuer Ideen. Ein spielerisches Beachten des Sequenzialitäts-Prinzips (s. Oevermann et al., 1979; Oevermann et al., 1980) kann eine förderliche Wirkung für die EntdeckungsHeuristik des Kodierens mit sich bringen“ (ebd., S. 261). Parallel zum Generieren von Kodes werden unterschiedlichste Gedanken als Memos dokumentiert wie z. B. erste Assoziationen zu Theorien. Eventuell lassen sich dabei bereits erste theoretische Kodes formulieren, die jedoch zugunsten einer umfassenden textnahen Analyse erst zu einem späteren Zeitpunkt in den Analyseprozess einbezogen werden (vgl. Strauss \& Corbin, 1996, S. 50) und dann auch zu einer tiefgründigen Auseinandersetzung mit wissenschaftlich abgesicherten Theorien und Modellen führen können. Anhang I im elektronischen Zusatzmaterial zeigt beispielhaft einen mit MAXQDA offen kodierten Textausschnitt. 


\section{Axiales Kodieren}

Idealtypisch sollen gemäß dem Kodierparadigma Zusammenhänge zwischen (ursächlichen) Kontextbedingungen, (Handlungs- und interaktionalen) Strategien und Konsequenzen im Hinblick auf das zentrale Phänomen herausgearbeitet und als Handlungsmodell dargestellt werden. Doch dieses von Strauss (1991) bzw. von Strauss und Corbin (1996) vorgegebene Schema stößt an seine Grenzen (vgl. Breuer et al., 2017, S. 290), beispielsweise wenn zirkuläre Strukturen in komplexen Wechselbeziehungen oder nicht aufzulösende Henne-Ei-Problemstellungen vorliegen (vgl. Hein, 2016, S. 179). Auch in der vorliegenden Studie lässt sich nicht jedes Phänomen mit dem Kodierparadigma erklären, geschweige denn, dass Kategorien, Konzepte und Kodes in der vorgegebenen Systematik als gemeinsames Ganzes dargestellt werden können.

Basis für das axiale Kodieren sind Kodes und Konzepte, die sich zu übergeordneten Sinnstrukturen, den Kategorien, gruppieren lassen. Anhang J1 im elektronischen Zusatzmaterial zeigt exemplarisch einen Ausschnitt aus der frühen Entwicklung von Kodes zu Kategorien, bei dem es im weiteren Datenauswertungsprozess zu vielfachen Umbenennungen, Neuzuordnungen und letztendlich zu einer neuen, feiner untergliederten und komplexeren Kodiersystematik kommt. Zudem veränderten sich Anzahl und Gewichtung der Kodes infolge der interviewübergreifenden iterativen Sequenzanalyse bei bereits ausgewerteten Interviews sowie infolge einer vertiefenden, deutlichen Abgrenzung der Kodes gegenüber Aussagen, die auch auf KE zutreffen.

Für die im elektronischen Zusatzmaterial in Anhang J1 dargestellte Momentaufnahme im axialen Kodierprozess, die ein frühes Auswertungsstadium abbildet, erscheinen die auf Präkonzepten basierenden vier Teilfragestellungen (Abschn. 2.3) noch anwendbar. Dies ist zu einem späteren Zeitpunkt, bei dem die Datenerweiterung und -analyse weiter fortgeschritten ist, nicht mehr der Fall. Denn aus dem darauffolgenden Datenauswertungsprozess gehen nicht nur mehr, sondern vor allem neue Erkenntnisse hervor. In der Folge zeigt sich das Beibehalten dieser Viergliederung, die aus der Analyse des Ist-Zustands präkonzeptionell hervorgegangen und somit nur als vorübergehende Ausgangssituation aufzufassen ist, nicht zielführend für das Beantworten der zentralen Fragestellung. Anhang J2 im elektronischen Zusatzmaterial zeigt diese Veränderung als weitere Momentaufnahme mitten in der axialen Kodierung auf. Deutlich wird, dass präkonzeptionell entstandene Strukturen eine eingeschränkte Sicht abbilden und als verzerrter Ausgangspunkt zu betrachten sind. Einerseits konkretisieren sie den Segen für den Forschungsbeginn, der per se einer Entscheidungs- und Bewertungswillkür unterworfen ist, so dass sie andererseits einen Forschungsfluch verkörpern und kontinuierlich auf den Prüfstand 
zu stellen sind. Präkonzeptionell entstandene Strukturen haben sich dementsprechend ununterbrochen im iterativen Forschungsprozess neuen Erkenntnissen zu unterwerfen, was sich $\mathrm{u}$. a. im axialen Kodierprozess zeigt.

Das Anwenden des in Tabelle 3.1 (Abschn. 3.4) skizzierten Konzept-IndikatorModells verschafft Transparenz für den Kodier-Prozess und verdeutlicht, auf welche intellektuelle Figur sich der Forscher bezieht.

\section{Selektives Kodieren}

Beim selektiven Kodieren werden die aus der axialen Kodierung hervorgegangenen relationalen Bezüge, die zwischen den kontextgebundenen und kausalen Konsequenzen bestehen, herausgearbeitet. Dabei lässt sich im methodologischen Lehrbuchdenken, jedoch nicht immer im realen Forschungsalltag, eine Kernkategorie identifizieren, die ein einziges zentrales Phänomen zum Vorschein bringt, dem sich alle weiteren Kategorien hierarchisch zu- und unterzuordnen haben (Strauss, 1991, S. 63; Strauss \& Corbin, 1996, S. 94; kritisch diskutiert z. B. bei Breuer et al., 2019, S. 132). Eine derart passgenaue Struktur herauszuarbeiten gleicht einem Geduldspiel, das sich durch mentales Jonglieren vielseitiger Gedankenimpulse auszeichnet und ein kontinuierliches Überarbeiten sowie Neuausrichten in der datenanalytischen Vorgehensweise mit sich bringt. Selbst wenn sich eine Kernkategorie nicht identifizieren lassen sollte, besteht die Zielsetzung, eine ganzheitlich-konsistente Systematik abzubilden, deren logischer Sinnzusammenhang zur Beantwortung der zentralen Forschungsfrage dient. Das Ergebnis kann daher beispielsweise ebenso eine zusammenhängende Theorie, ein kohärentes theoretisches (Prozess-)Modell oder eine Typologie-Systematik sein - jeweils mit begrenzter Reichweite, die sich aus den Rahmenbedingungen zur zentralen Forschungsfrage bemisst (vgl. z. B. Breuer et al., 2019, S. 287 ff.).

Die Darstellung des zeitlich nacheinander erfolgenden Dreischritts von offenem, axialem und selektivem Kodieren ist ebenfalls eine idealtypische Darstellung. In der Regel passieren die drei Teilschritte im iterativen Kodierprozess, da es ,sinnvoll sein [kann], aus Phasen des Axialen oder des Selektiven Kodierens wieder zurückzukehren zur Phase des Offenen Kodierens oder auch - nach dem Prinzip des Theoretical Sampling - neue Schritte der Datenerhebung zu unternehmen: Auf dem Hintergrund des bisherigen Verständnisses und der Erwartungen einer Forschenden hat sie u. U. einen interessanten und neuartigen theoretischen Aspekt entdeckt - und sie bemühe sich nun, dazu weitere passende empirische Phänomene oder Fälle zu finden, die ihre in Entwicklung befindliche Theorie erweitern, differenzieren oder modifizieren können“ (Breuer et al., 2017, S. 287; vgl. auch Flick, 2017, S. 387 ff.). 
Bis hierher wurde die in dieser Untersuchung übergreifend angewandte RGTKodiermethodik vorgestellt. Im Folgenden wird ausgeführt, weswegen und auf welche Weise darüber hinaus als Denkfigur ein metaphernanalytischer Auswertungsansatz integriert und das gesamte Datenmaterial auch qualitativ inhaltsanalytisch ausgewertet wird.

\subsubsection{Systematische metaphernanalytische Datenauswertung}

Während einer der ersten Interviewauswertungen fiel auf, dass relativ viele Memos und Kodes sprachliche Muster in den Schilderungen der befragten Person thematisieren bzw. auf diese hinweisen. Daher sollte diese Transkription dahingehend profund analysiert werden. Nach intensiver Literaturrecherche erschien hierfür ein systematisches metaphernanalytisches Verfahren optimal geeignet. Im ersten Schritt erfolgte hierzu eine solide Einarbeitung via Literatur-Selbststudium und Video-Tutorials. Die Entscheidung fiel daraufhin auf die Analysiersystematik nach Schmitt (2017). Ein bei ihm absolvierter Methodenworkshop für systematische Metaphernanalyse (vgl. Anhang B im elektronischen Zusatzmaterial) erwies sich als ergiebig und zielführend, da Auszüge der Interviewtranskription vom Experten angeleitet analysiert und die genaue weitere Vorgehensweise vermittelt wurde.

Bei der systematischen Metaphernanalyse wird das Transkript in seine metaphorischen Bestandteile zerlegt und auf das Vorhandensein von Mustern hin analysiert. Hierbei werden sowohl hermeneutische Feinanalysen der einzelnen Wörter vorgenommen als auch der Bezug von Wörtern zueinander systematisch verglichen. Hierauf aufbauend lassen sich die durch metaphorische Konzepte beschreibbaren Einstellungen und Handlungen rekonstruieren. Dabei werden zwei Arbeitsschritte parallel vollzogen: das Herausarbeiten, welche Redewendungen welchen Zielbereich treffen, und das Identifizieren der Konzepte, die darin enthalten sind. In einem Folgeschritt wird das Gesammelte sortiert. Zu einem späteren Zeitpunkt lassen sich die dabei gewonnenen Erkenntnisse mit der RGT-Kodiersystematik triangulieren.

Zielbereich der Metaphorisierung ist bei dem o. g. Interview das Konzeptverständnis der befragten Person zu Social Entrepreneurship. Im Ergebnis lässt sich hierbei ein Bruch herausarbeiten, da mehrere Konzepte nicht miteinander vereinbar sind, sie keine innere Konsistenz aufweisen. Beispielsweise fehlt ein Konzeptansatz, der das Soziale und das Unternehmerische miteinander verbindet. Scheinbar bleibt die IP durch die Kraft verankerter Schemata fest in 
ihren unverbundenen Konzepten verhaftet; in jedem Fall besteht keine ausdifferenzierte, harmonisierende Konzeptualisierung, keine Verbindungsmetaphorik und auch keine Balancemetaphorik. Bei dieser IP spiegelt das Unternehmerische beim sozialunternehmerischen Gründen und Handeln einerseits den Prozess des „Bauens“ wider, bei dem die Tätigkeit einem „mechanischen“ Prozess, dem Konstruieren von „Maschinen“ und von „Gebäuden“ gleicht und bei dem unvorhergesehene Baubarrieren auftreten (siehe Zitatbeispiele in der Endnote ${ }^{14}$ ). Ebenso verkörpert das Unternehmerische beim sozialunternehmerischen Handeln Bewegungsenergie und benötigt Antrieb (siehe Zitatbeispiele in der Endnote ${ }^{15}$ ). Andererseits gleicht das Soziale beim sozialunternehmerischen Handeln ab dem SEO-Gründungsprozess dem Zeugen eines Kindes: Ein Baby entsteht und es passiert organisches Wachstum, bei dem die Entwicklung des Babys erlebt und es auf dem Weg seiner reifenden Entwicklung beschützend begleitet wird (siehe Zitatbeispiele in der Endnote ${ }^{16}$ ), wobei zwischenmenschliche Kontaktgestaltung hohe Bedeutung erfährt (siehe Zitatbeispiele in der Endnote ${ }^{17}$ ). Insoweit gleicht das sozialunternehmerische Handeln einem Leben in zwei Welten, geprägt von Widerstand und (Ab-)Brüchen (siehe Zitatbeispiele in der Endnote ${ }^{18}$ ): Das Baby muss im Laufe seines Lebens aus Fehlern dazulernen, aber auf eine Maschine soll von Anfang an Verlass sein.

Weitere Sequenzen aus anderen Interviews wurden im Verlauf ebenfalls anlassbezogen zu verschiedenen Zielbereichen metaphernanalytisch ausgewertet. Dabei konnte für viele IP herausgearbeitet werden, dass weder eine Verbindungsmetaphorik noch eine Balancemetaphorik zum SE-Konzeptverständnis besteht. In Triangulation mit einem qualitativen inhaltsanalytischen Verfahren sowie der RGT-Kodiersystematik ließ sich dabei ein Phänomen ermitteln, das zur Beantwortung der Forschungsfrage beiträgt. Es wird mit seinen für Forschung und Praxis relevanten Aspekten in Kapitel 8 näher dargestellt.

\subsubsection{Qualitativ inhaltsanalytische Datenauswertung}

Während der Interviews teilten alle IP mit, welche Anforderungen sie selbst an einen "Social Entrepreneur" stellen und welche grundlegenden, allgemeingültigen Anforderungen an „Social Entrepreneurship“ sie dabei zugrunde legen. Im Rahmen der Datenauswertung sollten die Antworten der Befragten daraufhin analysiert, klassifiziert und bewertet werden, inwieweit die Befragten selbst die von ihnen genannten Anforderungen erfüllen und was dies hinsichtlich der eigenen Arbeitsbelastung bedeuten kann. Für diese Teilfragestellung eignet sich 
demnach ein evaluatives Verfahren, das in Anlehnung an die evaluative qualitative Inhaltsanalyse nach Kuckartz (2016, S. 123 ff.) durchgeführt wird. Dabei werden bei der initiierenden Textarbeit Indikatoren herausgearbeitet und passgenau einem Bewertungskriterium zugeordnet. Beispielsweise wurden in dieser Untersuchung die Indikatoren „Profitorientierung ist genauso wichtig wie soziale Orientierung“ und „Profit hat sich dem Sozialen immer unterzuordnen“ dem Kriterium „Profitorientierung“ zugeordnet. Damit sich sowohl sämtliche Kriterien bis zur Indikatorenebene fallbezogen als auch einzelne Kriterien im Fallvergleich auswerten lassen, wurde hierzu eine Matrixstruktur gebildet. In die Matrixstruktur wurden für jeden Befragten die Ausprägungen sämtlicher Indikatoren nach der klassischen Ampelsystematik bewertet. Dabei wurde die Übereinstimmung der benannten Anforderungen mit dem eigenen sozialunternehmerischen Agieren mit Grün gekennzeichnet, eine eingeschränkte, nur teilweise gegebene Übereinstimmung mit Gelb und keine Übereinstimmung mit Rot. Das Resultat stellt eine einfache kategorienbasierte Auswertung dar (ebd., S. 125). Die in den 35 Interviews identifizierten Kategorien und deren Evaluation auf Indikatorenebene sind wiedergegeben in der tabellarischen Darstellung im elektronischen Zusatzmaterial im Anhang K. Aus dieser evaluativen qualitativen Inhaltsanalyse lassen sich wichtige Erkenntnisse ableiten. So erheben zum Beispiel elf Befragte innerhalb der Kategorie „Problembewältigung“ einen Problemlösungsanspruch für jeden SE, allerdings haben zehn der Befragten die von ihnen identifizierten (sozialen bzw. sozioökologischen) Probleme nicht und eine Person nur eingeschränkt gelöst.

Im Anschluss an die evaluative qualitative Inhaltsanalyse wurde der gesamte innerhalb der Matrixstruktur herausgearbeitete Erkenntnisgewinn dem RGTKodierprozess zugeführt und bei der weiteren Kodierarbeit berücksichtigt. Denn „[a]uf die Kategorienbildung aufbauende Zusammenhangsanalysen, z. B. in Form von Kreuztabellen, erlauben es, im Anschluss an die evaluative Codierung Vermutungen über Zusammenhänge explorativ zu untersuchen“ (Kuckartz, 2016, S. 124).

Der im Rahmen der evaluativen qualitativen Inhaltsanalyse herausgearbeitete, für Forschung und Praxis relevante Erkenntnisgewinn wird ebenfalls in Kapitel 8 bei der Ergebnisdarstellung und -diskussion genau beschrieben.

Darüber hinaus findet ein weiteres qualitativ inhaltsanalytisches Verfahren Anwendung. Anlass hierfür war das Identifizieren inhaltlich widersprüchlicher Schilderungen bei einer befragten IP, was auch in zwei RGT-KodierArbeitsgruppen Gegenstand der Diskussion war. Daher sollte der gesamte Text auf Widersprüchlichkeiten hin untersucht werden. Dies erfolgte in einer Variation der inhaltlich strukturierenden qualitativen Inhaltsanalyse nach Kuckartz (2016, S. 97 ff.). In der Abwandlung ergeben sich die Kategorien nicht ausschließlich 
aus dem Leitfaden oder aus der verbalisierten Fragestellung; dies würde den Auswertungsfokus zu sehr eingrenzen. Vielmehr wurden darüber hinaus Themen, die sich erst im Interview ergeben haben, beim Herausarbeiten von Haupt- und Subkategorien einbezogen. Daraufhin erfolgte eine tabellarische Gegenüberstellung. Bei der initiierenden Textarbeit wurden Markierungen im Text vorgenommen und Memos notiert. Bereits vorhandene Memos, die zuvor im Rahmen der RGT gebildet worden sind, wurden ebenfalls einbezogen. Zur Analyse wurde der Text als Hardcopy zerschnitten. Vergleichbar mit einem Memory-Spiel wurde im Transkript nach dem passenden zweiten Segment gesucht. Dieses ist allerdings nicht deckungsgleich mit dem ersten Segment, sondern steht in seiner inhaltlichen Aussage im Widerspruch zu diesem - sozusagen als Negativ. Auf diese Weise können Segmente mit nicht nachvollziehbarem Aussagegehalt sowie mit Widersprüchlichkeiten in der gesamten Transkription systematisch gegenübergestellt und mit dazu verfassten Memos aufbereitet werden. Dabei findet ein kontinuierlicher Vergleich aller Segmente zueinander statt, was ein relativ aufwendiges, aber auch aussagekräftiges Verfahren darstellen kann. Die dabei herausgearbeiteten Erkenntnisse lassen sich bei der weiteren RGT-Kodierarbeit gut berücksichtigen, zumal Widersprüchlichkeiten ein Konsistenzmerkmal darstellen können (vgl. Kruse, 2014, S. 57). Im Rahmen der weiterführenden Analyse zeigt sich nicht nur bei dieser IP, sondern für nahezu alle IP, dass die Einstellung zu ,social“ und ebenso die Einstellung zu ,entrepreneurial“" jeweils positive und negative Anteile enthalten. Dies unterscheidet sich von den gängigen Darstellungen in der Fachliteratur. Das in Triangulation mit der RGT-Kodiersystematik entwickelte Muster lässt sich grafisch darstellen (siehe Anhang L im elektronischen Zusatzmaterial) und wird mit den für Forschung und Praxis relevanten Aspekten ebenfalls im achten Kapitel bei der Ergebnisdarstellung gewürdigt. Dabei bestehen im Erkenntnisgewinn einige Überschneidungen zur metaphernanalytischen Auswertung.

Der dargestellten Planung und Durchführung der Datenauswertung folgt nun eine methodenkritische Reflexion auf Basis der Gütekriterien qualitativer Forschung.

\subsubsection{Methodenkritische Reflexion zur Datenauswertung}

Die transparente Darstellung der Dokumentation gewährleistet die Nachvollziehbarkeit der Erkenntnisse und ermöglicht es, den Erkenntnisgewinn nachvollziehbar zu machen. Hierbei sind weitere Aspekte methodenkritisch zu reflektieren: 


\section{Datenauswertungssoftware MAXQDA}

Mein Arbeiten mit der Auswertungssoftware MAXQDA erwies sich für sämtliche der in Abschnitt 3.3.2 beschriebenen Zielsetzungen als sehr hilfreich. Neben einem gewissenhaften, effektiven wie effizienten Anwenden der gewählten Verfahren zur Datenauswertung verhalf mir die Software-Anwendung, im komplexen Prozessgeschehen einen strukturierten Überblick beizubehalten.

Darüber hinaus zeigte sich ein weiterer Anwendungsnutzen: Im Rahmen der Kodier-Arbeitsgruppen wurde das kontextgebundene Erfassen von Memos zur Reflexion der eigenen Rolle als Forscher und zum Bedeutungsgehalt von Forschungsstandards gewährleistet und sowohl innerhalb der Arbeitsgruppen als auch in Supervisionssitzungen, Doktorandenkolloquien und Fortbildungsworkshops vertiefend aufgegriffen. Dies entspricht einem wichtigen Aspekt der sog. reflexiven Offenheit, der im Rahmen der methodenkritischen Reflexion qualitativer Forschungsprojekte ein hoher Stellenwert eingeräumt wird (vgl. Kruse, 2014, S. 41; Bohnsack, 2014, S. 24 ff.; Breuer et al., 2019, S. 369 ff.).

\section{Intersubjektivität und Inter-Koder-Agreement}

Den zentralen Bedeutungsgehalt von Subjektivität in der RGT habe ich bereits erläutert: Als Forscher bin ich ein Forschersubjekt und interagiere mit anderen Forschersubjekten, meinen Kodier-Arbeitsgruppen, sowie mit meinen Befragten, den Forschungssubjekten. Subjektivität bzw. die methodenkritische Reflexion zur Nachvollziehbarkeit und Vergleichbarkeit der Interaktionsprozesse zwischen den an der Studie beteiligten Subjekten erhebt damit den Anspruch eines Gütekriteriums qualitativer Forschung (vgl. z. B. Breuer, 2009; 2010; Breuer, Muckel \& Dieris, 2019; Steinke, 1999; 2007; 2013; Denzin, 2013). Während der gesamten Datenauswertung konnte ich mit meinen Kodier-Arbeitsgruppen den Einsatz mehrerer Forscher sicherstellen. Für das kontinuierliche Reflektieren des Arbeitsverständnisses nutzte ich auch bei dieser Forscherperson-Triangulation eine selbst modifizierte Leitstruktur nach Breuer (2018), die im elektronischen Zusatzmaterial im Anhang $M$ wiedergegeben ist.

Bei der Metaphernanalyse wurden ebenfalls mehrere Kodier-Arbeitsgruppen zur Auswertung herangezogen. Kodierer verstehen sich auch bei dieser Auswertungsmethode als Forschersubjekte, die ihre in das Auswertungsmaterial hineingetragene Metaphorik durch ein strukturiertes Vorgehen systematisch aufarbeiten, mit der Zielsetzung, sich der eigenen subjektiven Beeinflussung gewahr zu sein und das konzeptionelle Regelwerk der Metaphernanalyse anwenden zu können.

Im Gegensatz zu offeneren textanalytischen Auswertungsverfahren wie der RGT, sollten bei qualitativen Inhaltsanalysen mehrere Textteile zur Überprüfung der Verfahrensstabilität im Sinne der Intra-Koder-Reliabilität erneut kodiert werden 
(Mayring, 2020, S. 499), was ich im Rahmen der Überprüfung meines Kategoriensystems mehrfach gewährleisten konnte. Dabei zeigte sich eine Übereinstimmung der Kodier-Ergebnisse. Eine hohe Übereinstimmung zeigte sich ebenfalls in der Reflexion in einer Arbeitsgruppe, in der eine zweitkodierende Person Textauszüge im Sinne der Auswertungsobjektivität (ebd.) eigenständig bearbeitet hat. Die dabei aufgetauchten Inkongruenzen haben wir gemeinsam klärend reflektiert. Auf diese Weise konnte ich eine Kongruenz in den Auswertungsergebnissen sicherstellen. Dieses für qualitative Inhaltsanalysen relevante Inter-Koder-Agreement (vgl. z. B. Fenzl \& Mayring, 2017; Haberfellner \& Fenzl, 2017) besteht sowohl für Kategorien der SE-Anforderungen-Matrix (Anhang $\mathrm{K}$ im elektronischen Zusatzmaterial, hervorgegangen aus der evaluativen qualitativen Inhaltsanalyse) als auch für Kategorien der Widerspruch-Analyse (hervorgegangen aus der inhaltlich strukturierenden Inhaltsanalyse).

\section{Methoden-Triangulation}

Da ich meinen Untersuchungsgegenstand nicht nur mit der RGT-Kodiersystematik, sondern auch mit der systematischen Metaphernanalyse sowie qualitativen inhaltsanalytischen Verfahren ausgewertet habe, konnte ich, wenngleich begrenzt, mit dieser Verknüpfung eine Methoden-Triangulation sicherstellen. Die Vereinbarkeit der methodischen Vorgehensweisen habe ich in diesem Kapitel beschrieben. Dennoch ist kritisch anzumerken, dass das metaphernanalytische Vorgehen trotz der intensiven Einarbeitung nur auszugsweise und damit lückenhaft vorgenommen wurde, was die Auswertungsqualität beeinträchtigen kann. Somit ist davon auszugehen, dass über die gewonnenen Erkenntnisse hinaus weitere metaphorische Muster unentdeckt geblieben sind.

\section{Theoretical Sorting}

Bereits bei der Datenerhebung in Abschnitt 3.2 habe ich aufgezeigt, dass während des gesamten theoretischen Samplings forschungsfragenrelevante Entdeckungen auf den Prüfstand gestellt werden. Via Falsifikation wird einer sich entwickelnden Theorie widersprochen, und auch in dieser Studie habe ich nicht nach einer Theoriebestätigung gefahndet. Bei der RGT-Kodierung kann ich mit dem Herausarbeiten des Erkenntnisgewinns direkt aus dem Datenmaterial zwar sicherstellen, dass die Theoriebildung datenbasiert erfolgt. Dabei ist allerdings zu berücksichtigen, dass der wissenschaftliche Erkenntnisgewinn abhängig ist von der die Erkenntnis hervorbringenden Person. Dies widerspricht der Objektivitätsprämisse quantitativer Forschung, zumal die Zutaten für diesen Wirkmechanismus zwangsläufig aus einem Cocktail an Erfahrungswerten und Abstraktionsvermögen von mir und den wenngleich sehr interdisziplinär gestalteten Kodier-Arbeitsgruppenmitgliedern bestehen müssen. 
Die generierten theoretischen Memos dienen dazu, Gedanken zum hierarchischen Netz von Konstrukten in das Auswertungsmaterial einzubinden. Dabei habe ich die theoretischen Memos kontinuierlich erweitert, verändert und bei neu begründeten Vorstellungen zur Ausprägung eines hierarchischen Netzes von Konstrukten ggf. wieder verworfen. Das so entstandene Theoretical Sorting ist ein wichtiger Baustein für die spätere Theorieentwicklung (vgl. Strübing, 2008, S. 34). Gleichwohl wurden innerhalb der Kodier-Arbeitsgruppen vielseitige theoretische Memos an das Datenmaterial herangetragen, wobei ich das Theoretical Sorting limitieren und den Sättigungsprozess somit abbrechen musste. Dabei musste ich auch im Kodierprozess Limitationen vornehmen. Hier habe ich mich an die Empfehlung von Breuer (2018) gehalten, ab Beginn einer jeden fallbezogenen Auswertung sowohl im alleinigen als auch im Gruppen-Auswertungsprozess kleinschrittig und streng sequenziell zu kodieren. Dabei haben die aufgedeckten Muster einen umfangreichen Interpretationsspielraum eröffnet. Die theoretische Relevanz der Muster musste ich dementsprechend begründend herausarbeiten, andernfalls Kodierungen neu überdenken bzw. verwerfen. Dies hatte Nachkodierungen bereits kodierter Daten aufgrund neu gefundener Deutungshintergründe zur Folge, was dem zirkulären Arbeitsverständnis in der RGT entspricht.

\section{Validität}

Validität hat in der qualitativen Forschung einen eigenständigen Bedeutungsgehalt. Die Validität der Datenauswertung ist abhängig von der Qualität der Transkription und von der Tiefgründigkeit der regelgeleiteten Auswertung des Datenmaterials. Dabei ist dem Authentizitätsgebot Folge zu leisten (vgl. Steinke, 2007, S. 180 ff.; m. V. a. Guba \& Lincoln, 1989, S. 245 ff.; Manning, 1997), wonach sämtliche erhobenen Daten hinsichtlich des subjektiven Relevanzsystems der Befragten auszuwerten sind. Kritisch ist daher noch einmal anzumerken, dass die von den IP erhaltenen Materialien sowie weitere recherchierte Dokumente wie z. B. Videos und Artikel in Fachzeitschriften nicht in Gänze und systematisch kodiert werden konnten. Dies hätte den Rahmen der machbaren Auswertung gesprengt. Allerdings habe ich diese Begleitmaterialien auswertungsunterstützend herangezogen, so wie oben beschrieben. Das theoretische Sampling übernimmt dabei die Funktion eines Vehikels für das Generieren von Konsistenz (vgl. Corbin \& Strauss, 1990, S. 420 ff.); Sinnstrukturen werden als „Indikatoren, Konzepte und Kategorien konsistent miteinander verglichen“ (Steinke, 1999, S. 75). Dies kann dazu beitragen, Störvariablen zu identifizieren und zu eliminieren, was der internen Validität zugutekommt.

Einerseits schaffe ich mit dem in dieser Studie gewählten Forschungsdesign die empirisch abgesicherte Basis dafür, dass die Untersuchungsergebnisse prinzipiell auf weitere Social Entrepreneure und deren Lebenskontexte anwendbar sind, so 
dass hierbei eine ökologische Validität gegeben sein sollte. Andererseits ist kritisch anzumerken, dass es mir aus Ressourcengründen nicht für jedes Interview und ggf. nicht für sämtliche Passagen möglich war, Validierungsgespräche mit den Befragten zu führen. Den Bedeutungsgehalt von kommunikativer Validierung für eine Feedbacksicherung zu meinem Verständnis und meinen daraus abgeleiteten Maßnahmen habe ich bereits in Abschnitt 3.2.7 erläutert. Dabei bezog ich nach der Interviewdurchführung erhaltene Erläuterungen der Befragten in die Datenauswertung ein. Mit mehreren Befragten reflektierte ich gemeinsam die Gültigkeit meiner Datenauswertung und meine damit verbundenen Interpretationen, was einem feinfühligen Umgang mit den Daten im Auswertungsprozess dienlich ist und sich somit auch positiv auf die theoretische Sensitivität auswirkt (vgl. Breuer et al., 2019, S. 160 ff.; im Hinblick auf die kommunikative Validierung siehe auch Terhart, 1981, S. 772).

\section{Supervision, Intervision und Expertenberatung}

Während der Datenauswertung habe ich Kodier-Arbeitsgruppentreffen auch zur Selbstreflexion genutzt, wenn bei mir Indikatoren für Sympathie- bzw. AntipathieEffekte gegenüber den Befragten zutage getreten sind. Beide Effekte traten im Laufe der Interviews auf und kamen zum Teil auch bei den Mitgliedern der Kodier-Arbeitsgruppentreffen zum Vorschein. Sämtliche aufgedeckte Besonderheiten wurden arbeitsgruppenintern kommunikativ validiert in Form von regelmäBigen Intervisionssequenzen, um die Professionalität in der Auswertungsqualität zu wahren. Entsprechende Ausführungen wurden in Memos festgehalten und nachreflektiert.

Dennoch ist kritisch anzumerken, dass Kodier-Arbeitsgruppenteilnehmer nicht an Supervisionssitzungen teilgenommen haben und dass aus Ressourcengründen nicht für jedes Interview eine Supervisionssitzung absolviert wurde, so dass blinde Flecken verborgen geblieben sein können und dies auch zu erwarten ist.

Last, not least hat die aktive Kontaktgestaltung zu den Experten der absolvierten Methoden-Workshops über die absolvierten Kurse hinaus einen hohen Stellenwert für das Reflektieren der Methodenanwendung. In Telefonaten und per E-Mail erhielt ich ein Feedback zu meiner Vorgehensweise und Antworten auf Spezialfragen, die nur von Experten beantwortet werden konnten und dankenswerterweise auch stets zielführend beantwortet wurden.

Zur Nachvollziehbarkeit der Qualitätssicherung ist der einleitend bereits erwähnte, standardisierte Forschungsablauf im Überblick als Workflow in Abbildung 3.1 (Abschn. 3.1) wiedergegeben. 
Mit den methodologischen Ausführungen in diesem Kapitel wurde die empirisch notwendige Basis geschaffen und das Güteverständnis aufgezeigt für die nun folgende Ergebnisdarstellung und -diskussion.

\section{Literatur- und Quellenverzeichnis}

Böhm, A. (2013). Theoretisches Codieren: Textanalyse in der Grounded Theory. In: U. Flick, E. v. Kardorff und I. Steinke (Hrsg.), Qualitative Forschung. Ein Handbuch (10. Auflage). Reinbek bei Hamburg: Rowohlt Taschenbuch, S. 475-485.

Bohnsack, R. (2014). Rekonstruktive Sozialforschung Einführung in qualitative Methoden (9., erw. Auflage). Opladen: Budrich.

Breuer, F. (Hrsg.) (1996). Qualitative Psychologie. Grundlagen, Methoden und Anwendungen eines Forschungsstils. Opladen: Westdeutscher Verlag.

Breuer, F. (1999). Probleme human- und sozialwissenschaftlicher Erkenntnismethoden: Viel Verwirrung - einige Vorschläge. In: N. Groeben (Hrsg.), Zur Programmatik einer sozialwissenschaftlichen Psychologie. Band 1, Metatheoretische Perspektiven; 2. Halbband: Theoriehistorie, Praxisrelevanz, Interdisziplinarität, Methodenintegration. Münster: Aschendorff, S. 193-309.

Breuer, F. (2009). Reflexive Grounded Theory. Eine Einführung für die Forschungspraxis. Wiesbaden: VS Verlag für Sozialwissenschaften.

Breuer, F. (unter Mitarbeit von B. Dieris und A. Lettau) (2010). Reflexive Grounded Theory. Eine Einführung für die Forschungspraxis (2. Auflage). Wiesbaden: VS Verlag für Sozialwissenschaften.

Breuer, F. (2018). Unveröffentlichte Präsentation zum Methodenworkshop „Reflexive Grounded Theory für Fortgeschrittene“ mit Vor-Ort-Ergänzungen durch den Dozenten. 30.11.-1.12.2018, Institut für Qualitative Forschung (INA), Berlin.

Breuer, F. Muckel, P. \& Dieris, B. (2017). Reflexive Grounded Theory. Eine Einführung in die Forschungspraxis (3., vollständig überarbeitete, erweiterte Auflage). Wiesbaden: Springer VS.

Breuer, F. Muckel, P. \& Dieris, B. (2019). Reflexive Grounded Theory. Eine Einführung in die Forschungspraxis (4., aktualisierte Auflage). Wiesbaden: Springer VS.

Charmaz, K. (2001). Grounded theory analysis. In: J. Gubrium und J. Holstein (Hrsg.), Handbook of interview research. Context \& Method. Thousand Oaks (USA) et al.: Sage, S. 675-694.

Corbin, J. \& Strauss, A. (1990). Grounded theory research. Procedures, canons and evaluative criteria. In: Zeitschrift für Soziologie, 19, S. 418-427.

Denzin, N. (2013). Symbolischer Interaktionismus. In: U. Flick, E. v. Kardorff und I. Steinke (Hrsg.), Qualitative Forschung. Ein Handbuch (10. Auflage). Reinbek: Rowohlt, S. 136150 .

Fenzl, T. \& Mayring, P. (2017). QCAmap: eine interaktive Webapplikation für Qualitative Inhaltsanalyse. In: Zeitschrift für Soziologie der Erziehung und Sozialisation, 37 (3), S. 333-340. 
Flick, U. (2017). Qualitative Sozialforschung. Eine Einführung (8. Auflage). Reinbek: Rowohlts Enzyklopädie.

Flick, U., Kardorff E. v. \& Steinke, I. (Hrsg.) (2013). Qualitative Forschung. Ein Handbuch (10. Auflage). Reinbek: Rowohlt.

Glaser, B. (2012). No Preconception: The Dictum. In: Grounded Theory Review, 2 (11, Dezember 2012).

Glaser, B. \& Holton, J. (2011). Der Umbau der Grounded Theory Methodology. In: G. Mey und K. Mruck (Hrsg.), Grounded Theory Reader (2., aktualisierte, erweiterte Auflage). Wiesbaden: VS Verlag für Sozialwissenschaften \& Springer Fachmedien, S. 137-162.

Glaser, B. \& Strauss, A. (1965). Awareness of Dying. Chicago (USA): Aldine.

Glaser, B. \& Strauss, A. (1967). The Discovery of Grounded Theory. Strategies for Qualitative Research. Chicago (USA): Aldine.

Glaser, B. \& Strauss, A. (1998). Grounded Theory. Strategien qualitativer Forschung. Bern (Schweiz) et al.: Huber.

Glaser, B. \& Tarozzi, M. (2011). Vierzig Jahre nach ,The Discovery“. Grounded Theory weltweit. In: G. Mey und K. Mruck (Hrsg.), Grounded Theory Reader (2., aktualisierte, erweiterte Auflage). Wiesbaden: VS Verlag für Sozialwissenschaften \& Springer Fachmedien, S. 53-67.

Guba, E. \& Lincoln, Y. (1989). Fourth generation evaluation. Newbury Park (USA): Sage.

Haberfellner, C. \& Fenzl, T. (2017). The utility value of research evidence for educational practice from the perspective of preservice student teachers in Austria. A qualitative exploratory study. In: Journal for Educational Research Online, 9 (2), S. 67-85.

Hein, R. (2016). Erfolg im Compliance Management. Konfliktfelder erkennen und bewältigen: Arbeits- und organisationspsychologische Anregungen. Wiesbaden: Springer Gabler.

Helfferich, C. (2011). Die Qualität qualitativer Daten. Manual für die Durchführung qualitativer Interviews (4. Auflage). Wiesbaden: VS Verlag.

Hussy, W., Schreier, M. \& Echterhoff, G. (2013). Forschungsmethoden in Psychologie und Sozialwissenschaften für Bachelor (2. Auflage). Berlin \& Heidelberg: Springer.

Kruse, J. (2014). Qualitative Interviewforschung. Ein integrativer Ansatz. Weinheim \& Basel (Schweiz): Beltz Juventa.

Kuckartz, U. (2016). Qualitative Inhaltsanalyse. Methoden, Praxis, Computerunterstützung (3. Auflage). Weinheim \& Basel (Schweiz): Beltz Juventa.

Lamnek, S. (2010). Qualitative Sozialforschung (5., überarbeitete Auflage). Weinheim \& Basel (Schweiz): Beltz.

Legewie, H. \& Schervier-Legewie, B. (1995). Im Gespräch: Anselm Strauss. In: Journal für Psychologie, 3 (1), S. 64-75.

Manning, K. (1997). Authenticity in Constructivist Inquiry: Methodological Considerations Without Prescription. In: Qualitative Inquiry, 3 (1), S. 93-115.

Mayring, P. (2016). Einführung in die qualitative Sozialforschung. Weinheim \& Basel (Schweiz): Beltz.

Mayring, P. (2020). Qualitative Inhaltsanalyse. In: G. Mey \& K. Mruck (Hrsg.), Handbuch Qualitative Forschung in der Psychologie (Band 2: Designs und Verfahren; 2., erweiterte und überarbeitete Auflage). Wiesbaden: Springer, S. 495-511.

Mey, G. (2000). Erzählungen in qualitativen Interviews: Konzepte, Probleme, soziale Konstruktionen. Sozialer Sinn. In: Zeitschrift für hermeneutische Sozialforschung, 1 (1), S. 135-151. 
Oevermann, U., Allert, T., Konau, E. \& Krambeck, J. (1979). Die Methodologie einer „objektiven Hermeneutik“ und ihre allgemeine forschungslogische Bedeutung in den Sozialwissenschaften. In: H.-G. Soeffner (Hrsg.), Interpretative Verfahren in den Sozial- und Textwissenschaften. Stuttgart: Metzler, S. 352-434.

Oevermann, U., Allert, T. \& Konau, E. (1980). Zur Logik der Interpretation von Interviewtexten. In: T. Heinze, H. Klusemann und H.-G. Soeffner (Hrsg.), Interpretation einer Bildungsgeschichte. Bensheim: Päd. extra, S.15-69.

Przyborski, A. \& Wohlrab-Sahr, M. (2014). Qualitative Sozialforschung. Ein Arbeitsbuch (4., erweiterte Auflage). München: Oldenbourg.

Reiser, H. \& Lotz, W. (1995). Themenzentrierte Interaktion als Pädagogik. Mainz: Grünewald. Robson, C. (1993). Real World Research. A Resource for Social Scientists and PractitionerResearchers. Oxford (Großbritannien) \& Cambridge (USA): Blackwell.

Robson, C. (2002). Real World Research. A Resource for Social Scientists and PractitionerResearchers (2., überarbeitete Auflage). Oxford (Großbritannien): Blackwell.

Rogers, C. (1985). Die nicht-direktive Beratung. Frankfurt: Fischer.

Schmitt, R. (2017). Systematische Metaphernanalyse als Methode der qualitativen Sozialforschung. Wiesbaden: Springer VS.

Schütze, F, (1977). Die Technik des narrativen Interviews in Interaktionsfeldstudien, dargestellt an einem Projekt zur Erforschung von kommunalen Machtstrukturen. Arbeitsberichte und Forschungsmaterialien, Universität Bielefeld, Fakultät für Soziologie, Nr. 1.

Steinke, I. (1999). Kriterien qualitativer Forschung. Ansätze zur Bewertung qualitativempirischer Sozialforschung. Weinheim: Juventa.

Steinke, I. (2007). Qualitätssicherung in der qualitativen Forschung. In: U. Kuckartz, H. Grunenberg und T. Dresing (Hrsg.), Qualitative Datenanalyse: computergestützt. Methodische Hintergründe und Beispiele aus der Forschungspraxis (2., überarbeitete, erweiterte Auflage). Wiesbaden: VS Verlag für Sozialwissenschaften, S. 176-187.

Steinke, I. (2013). Gütekriterien qualitativer Forschung. In: U. Flick, E. v. Kardorff und I. Steinke (Hrsg.), Qualitative Forschung. Ein Handbuch (10. Auflage). Reinbek: Rowohlt, S. 319-331.

Strauss, A. (1991). Grundlagen qualitativer Sozialforschung. Datenanalyse und Theoriebildung in der empirischen soziologischen Forschung. Aus dem Amerikanischen von Astrid Hildenbrand. Übergänge, Bd. 10. München: Wilhelm Fink (Original: Qualitative Analysis for Social Scientists, 1987, Cambridge: Cambridge University).

Strauss, A. \& Corbin, J. (1996). Grounded Theory. Grundlagen Qualitativer Sozialforschung. Weinheim: Beltz Psychologie Verlags Union.

Strübing, J. (2008). Grounded Theory. Zur sozialtheoretischen und epistemologischen Fundierung des Verfahrens der empirisch begründeten Theoriebildung (2., überarbeitete, erweiterte Auflage). Wiesbaden: VS Verlag für Sozialwissenschaften.

Strübing, J. (2014). Grounded Theory und Theoretical Sampling. In: N. Baur und J. Blasius (Hrsg.), Handbuch Methoden der empirischen Sozialforschung. Wiesbaden: Springer VS, S. 457-572.

Terhart, E. (1981). Intuition - Interpretation - Argumentation. Zum Problem der Geltungsbegründung von Interpretationen. In: Zeitschrift für Pädagogik, 27, S. 769-793.

Watzlawick, P., Beavin, J. \& Jackson, D. (1990). Menschliche Kommunikation. Formen, Störungen, Paradoxien (8. Auflage). Bern (Schweiz): Huber. 
Witzel, A. (1985). Das problemzentrierte Interview. In: G. Jüttemann (Hrsg.), Qualitative Forschung in der Psychologie. Grundfragen, Verfahrensweisen, Anwendungsfelder. Weinheim: Beltz, S. 227-255.

Witzel, A. (2000). Das problemzentrierte Interview. In: Forum Qualitative Sozialforschung (FQS), Online Journal vom Januar 2000, Bd. 1, Nr. 1. Abrufbar auf: http://www.qualit ative-research.net/index.php/fqs/article/view/1132/2519. Abgerufen am: 4. 8.2014.

Open Access Dieses Kapitel wird unter der Creative Commons Namensnennung 4.0 International Lizenz (http://creativecommons.org/licenses/by/4.0/deed.de) veröffentlicht, welche die Nutzung, Vervielfältigung, Bearbeitung, Verbreitung und Wiedergabe in jeglichem Medium und Format erlaubt, sofern Sie den/die ursprünglichen Autor(en) und die Quelle ordnungsgemäß nennen, einen Link zur Creative Commons Lizenz beifügen und angeben, ob Änderungen vorgenommen wurden.

Die in diesem Kapitel enthaltenen Bilder und sonstiges Drittmaterial unterliegen ebenfalls der genannten Creative Commons Lizenz, sofern sich aus der Abbildungslegende nichts anderes ergibt. Sofern das betreffende Material nicht unter der genannten Creative Commons Lizenz steht und die betreffende Handlung nicht nach gesetzlichen Vorschriften erlaubt ist, ist für die oben aufgeführten Weiterverwendungen des Materials die Einwilligung des jeweiligen Rechteinhabers einzuholen.

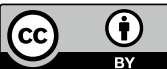

